J. Gancarzewicz, W. Mikulski and Z. Pogoda

Nagoya Math. J.

Vol. 135 (1994), 1-41

\title{
LIFTS OF SOME TENSOR FIELDS AND CONNECTIONS TO PRODUCT PRESERVING FUNCTORS
}

\author{
JACEK GANCARZEWICZ, WŁODZIMIERZ MIKULSKI \\ AND ZDZISŁAW POGODA
}

\section{Introduction}

In this paper we define some lifts of tensor fields of types $(1, k)$ and $(0, k)$ as well as connections to a product preserving functor $\mathscr{F}$. We study algebraic properties of introduced lifts and we apply these lifts to prolongation of geometric structures from a manifold $M$ to $\mathscr{F}(M)$. In particular cases of the tangent bundle of $p^{r}$-velocities and the tangent bundle of infinitesimal near points our constructions contain all constructions due to Morimoto (see [20]-[23]). In the cases of the tangent bundle our definitions coincide with the definitions of Yano and Kobayashi (see [31]). To construct our lifts and to study its properties we use only general properties of product preserving functors. All lifts verify so-called the naturality condition. It means that for a smooth mapping $\varphi: M \rightarrow N$ and for two $\varphi$-related geometric objects defined on $M$ and $N$ its lifts to $\mathscr{F}(M)$ and $\mathscr{F}(N)$ respectively are $\mathscr{F}(\varphi)$-related. We explain later the term $\varphi$-related for considered geometric objects.

In the presented paper we do not study problems of classifications of lifts.

A product preserving functor is a covariant functor $\mathscr{F}$ from the category of manifolds into the category of fibered manifolds such that $\mathscr{F}\left(M_{1} \times M_{2}\right)$ is equivalent to $\mathscr{F}\left(M_{1}\right) \times \mathscr{F}\left(M_{2}\right)$. In Section 1 we formulate properties of product preserving functors used in the present paper.

Let $\mathscr{F}$ be a product preserving functor. In Section 2 we recall lifts of vector fields and functions to $\mathscr{F}$. Lifts of vector fields was introduced by Koláŕ in [14]. They are parametrized by elements of so-called the Weil algebra $A=\mathscr{F}(\mathbf{R})$ associated to $\mathscr{F}$. Lifts of functions to $\mathscr{F}$ was studied by Mikulski in [17]. They depend on functions $\lambda: A \rightarrow \mathbf{R}$. The defined lifts verify the naturality condition.

Let $\varphi: M \rightarrow N$ be a smooth mapping. Vector fields $X, Y$ defined on $M$ and $N$ respectively are called $\varphi$-related if the following diagram

Received June 10, 1993. 


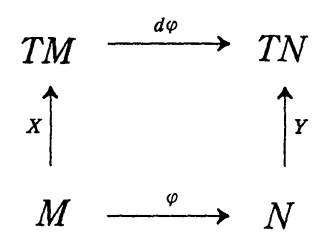

commutes. Two functions $f, g$ defined on $M$ and $N$ respectively are called $\varphi$-related if $f=g \circ \varphi$. In Section 2 we prove a few new and "nice" algebraic properties of introduced lifts which will be very useful in other sections.

In Section 3 we define lifts of tensor fields of type $(1, k)$. This family of lifts is parametrized by elements of the Weil algebra $A=\mathscr{F}(\mathbf{R})$ associated to $\mathscr{F}$. Each $a$-lift verifies the naturality condition. In this case for a smooth mapping $\varphi: M \rightarrow N$ two tensor fields $S, S^{\prime}$ of type $(1, k)$ defined on $M$ and $N$ respectively are called $\varphi$-related if the following diagram

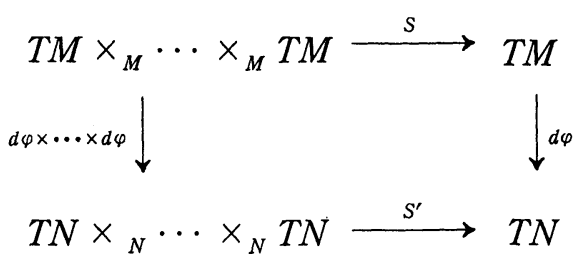

commutes.

We study algebraic properties of $a$-lift, where $a \in A$. Among $a$-lifts $S^{(a)}$ so called complete lift $S^{C}=S^{(1)}$, where 1 is the unity of $A$, is the most important. From the proved properties of $a$-lifts we deduce that for a tensor field $S$ of type $(1,1)$ and for a polynomial $W(t)$ we have $W\left(S^{C}\right)=(W(S))^{c}$ (see Proposition 3.2). It implies that for an almost complex structure (respectively an almost tangent structure, an $f$-structure) $S$ on $M$ its complete lift $S^{C}$ is an almost complex structure (respectively an almost tangent structure, an $f$-structure) on $\mathscr{F}(M)$ (see Corollary 3.3). Next we verify that for a tensor field $S$ of type $(1,1)$ on $M$ we have

$$
N_{S^{c}}=\left(N_{S}\right)^{C} \text {, }
$$

where $N_{S}$ is the Nijenhuis tensor of $S$ (see Proposition 3.4). From this properties we conclude that for an almost complex structure (respectively an almost tangent structure, an $f$-structure) $S$ on $M$ its complete lift $S^{C}$ is integrable if and only if so is $S$ (see Theorem 3.5). 
In Section 4 we study lifts of tensor fields of type $(0, k)$. This family of lifts is parametrized by linear functions $\lambda: A \rightarrow \mathbf{R}$ on the Weil algebra $A=\mathscr{F}(\mathbf{R})$ of $\mathscr{F}$. Each $\lambda$-lift satisfies the naturality condition. Analogously as for tensor fields of type $(1, k)$ two tensor fields $G, G^{\prime}$ of type $(0, k)$ are called $\varphi$-related if the following diagram

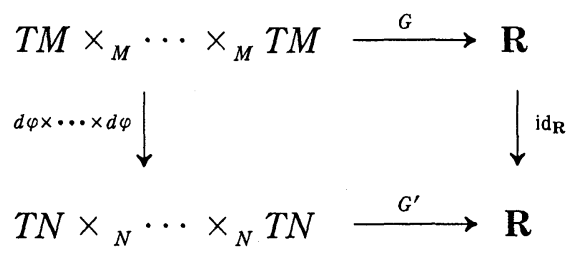

commutes.

Next analogously as in previous sections we prove some properties of $\lambda$-lifts of tensor fields of type $(0, k)$. Particularly, for a symmetric tensor $G$ on $M$ we calculate the signature of $G^{(\lambda)}$ as a function of the signature of $G$ and the signature of the symmetric form $A^{2} \ni(a, b) \rightarrow \lambda(a b) \in \mathbf{R}$ associated with $\lambda$. From the proved formula (see Proposition 4.5) we conclude immediately:

$G^{(\lambda)}$ is non-singular if and only if $G$ and the form $(a, b) \rightarrow \lambda(a b)$ are non-singular

$G^{(\lambda)}$ is positive definite if and only if $G$ and the form $(a, b) \rightarrow \lambda(a b)$ are positive definite.

We observe (Lemma 4.6) that there is no linear function $\lambda: A \rightarrow \mathbf{R}$ on the Weil algebra of $\mathscr{F}$ such that the associated form $A^{2} \ni(a, b) \rightarrow \lambda(a b) \in \mathbf{R}$ is positive definite instead of the identity functor $\mathscr{F}$ given by $\mathscr{F}(M)=M$ and $\mathscr{F}(\varphi)=\varphi$. It means that we cannot obtain a Riemannian metric on $\mathscr{F}(M)$ by $\lambda$-lifts of Riemannian metrics from $M$. To obtain a pseudo-Riemannian metric or an almost symplectic form on $\mathscr{F}(M)$ by a $\lambda$-lift of a pseudo-Riemannian metric or an almost symplectic form from $M$ we need to use a linear function $\lambda: A \rightarrow \mathbf{R}$ on the Weil algebra $A$ of $\mathscr{F}$ such that the associated symmetric form $(a, b) \rightarrow$ $\lambda(a b)$ is non-singular. In general, there is no function $\lambda$ with this property. We give a necessary condition for the existence of such a function $\lambda$ (see Proposition 4.7). We study properties of lifted (pseudo-)Riemannian metrics and (almost) symplectic forms in Section 6 .

In Section 5 we define a complete lift of a linear connection from $M$ to a linear connection on $\mathscr{F}(M)$ and we study its properties. The complete lift of linear connections verifies the naturality condition only for all embeddings $\varphi: M \rightarrow N$ of two $n$-dimensional manifolds. For linear connections $\nabla$ and $\nabla^{\prime}$ defined on $M$ and $N$ respectively we say that they are $\varphi$-related if for all $\varphi$-related vector fields $X$, 
$X^{\prime}$ and all $\varphi$-related vector fields $Y, Y^{\prime}$ the vector fields $\nabla_{X} Y$ and $\nabla_{X^{\prime}}^{\prime} Y^{\prime}$ are also $\varphi$-related.

We prove a "nice" formula for covariant derivations. If $\nabla$ is a linear connection on $M$, then its complete lift $\nabla^{C}$ is the unique linear connection on $\mathscr{F}(M)$ such that

$$
\nabla_{X^{(a)}}^{C} Y^{(b)}=\left(\nabla_{X} Y\right)^{(a b)}
$$

for all vector fields $X, Y$ on $M$ and all $a, b \in A$ (Proposition 5.6). From this we can conclude that the torsion and the curvature of $\nabla^{C}$ are the complete lifts of the torsion and the curvature of $\nabla$ (Proposition 5.6).

Lifts of generalized connections to a Weil functor were studied by Slovák [28].

At the end of this section we prove that lifts of tensor fields commute with the complete lift of linear connections (Proposition 5.11).

In Section 6 we study lifts of pseudo-Riemannian metrics and (almost) symplectic forms and we prove some standard properties on Riemannian metrics, Kählerian structures and the integrability of lifted almost symplectic structures. We can prolong such geometric structures to $\mathscr{F}(M)$ under the condition that there is a linear function $\lambda: A \rightarrow \mathbf{R}$ on the Weil algebra $A=\mathscr{F}(\mathbf{R})$ such that the associated symmetric form $A^{2} \ni(a, b) \rightarrow \lambda(a b) \in \mathbf{R}$ is non-singular. We recall that in general there is no function with this properties. In the case of the $r$-order tangent bundle such a function exists and in this case considering a suitable function $\lambda$ our propositions coincide with the corresponding results of Morimoto [22].

In the last section we formulate corresponding local expressions of lifted objects. $\mathscr{C}^{\infty}$

In the paper we always suppose that all manifolds and mappings are of class

\section{Product preserving functors}

First we recall the definitions of prolongation functor and product preserving functor.

Definition 1.1. A prolongation functor is a covariant functor $\mathscr{F}$ from the category of all manifolds and all mappings into the category of fibered manifolds satisfying the following conditions:

(1) For every manifold $M, \mathscr{F}(M)$ is a fibered manifold over $M$ and $\pi=\pi_{M}$ : $\mathscr{F} M \rightarrow M$ is its projection. For a point $x \in M$ we denote by $\mathscr{F}_{x}(M)=$ 
$\pi_{M}^{-1}(x)$ the fibre over $x$.

(2) The naturality condition. For every mapping $\varphi: M \rightarrow N$ of two manifolds $M, N$, for the induced mapping $\mathscr{F}(\varphi): \mathscr{F}(M) \rightarrow \mathscr{F}(N)$ the following diagram

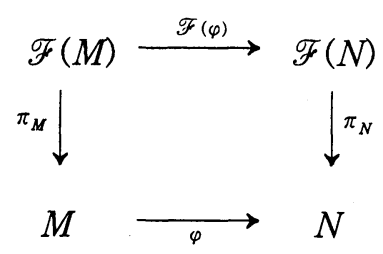

commutes.

(3) If $\varphi: M \rightarrow N$ is an embedding of two $n$-dimensional manifolds $M, N$, then for $x \in M$ the restriction $\mathscr{F}(\varphi)_{\mid \mathscr{F}_{x}(M)}: \mathscr{F}_{x}(M) \rightarrow \mathscr{F}_{\varphi(x)}(N)$ is a diffeomorphism.

(4) The regularity condition. If $\varphi_{t}: M \rightarrow N$ is a differentiable family of mappings, then $\mathscr{F}\left(\varphi_{t}\right): \mathscr{F}(M) \rightarrow \mathscr{F}(N)$ is a differentiable family of mappings.

Let us observe that for every fixed natural number $n$ the restriction of a prolongation functor to the category of $n$-dimensional manifolds and their embeddings is so-called a natural bundle (see [25]). Kolár and Solvák have proved that the reg. ularity condition is a consequence of conditions (1) and (2) of Definition 1.1 (see [15]).

This definition immediately implies:

(1) if $U \subset M$ is an open subset then we can identify $\mathscr{F}(U)$ with $\mathscr{F}(M)_{\mid U}$ by $\mathscr{F}(i): \mathscr{F}(U) \rightarrow \mathscr{F}(M)_{\mid U}$, where $i: U \rightarrow M$ is the inclusion;

(2) $\mathscr{F}\left(\mathbf{R}^{n}\right)$ is isomorphic with the trivial bundle $\mathbf{R}^{n} \times F$, where $F=\mathscr{F}_{0}\left(\mathbf{R}^{n}\right)$. The isomorphism $\Psi: \mathbf{R}^{n} \times F \rightarrow \mathscr{F}\left(\mathbf{R}^{n}\right)$ is given by $\Psi(x, y)=\mathscr{F}\left(\tau_{x}\right)(y)$, where $\tau_{x}: \mathbf{R}^{n} \rightarrow \mathbf{R}^{n}$ is the translation.

Every prolongation functor transforms immersions, submersions and embeddings into immersions, submersions and embeddings respectively (see [15]).

For two manifolds $M_{1}, M_{2}$ we denote by $\pi_{i}: M_{1} \times M_{2} \rightarrow M_{i}$ the standard projection on the $i$-th factor, where $i=1,2$.

Definition 1.2. A prolongation functor $\mathscr{F}$ is called a product preserving functor if for all manifolds $M_{1}, M_{2}$ the mapping

$$
\left(\mathscr{F}\left(\pi_{1}\right), \mathscr{F}\left(\pi_{2}\right)\right): \mathscr{F}\left(M_{1} \times M_{2}\right) \rightarrow \mathscr{F}\left(M_{1}\right) \times \mathscr{F}\left(M_{2}\right)
$$

is a diffeomorphism.

For a product preserving functor $\mathscr{F}$ we will always identify $\mathscr{F}\left(M_{1} \times M_{2}\right)$ 
with $\mathscr{F}\left(M_{1}\right) \times \mathscr{F}\left(M_{2}\right)$ by the deffeomorphism from the definition. After this identification we have

$$
\begin{gathered}
\mathscr{F}\left(f_{1} \times f_{2}\right)=\mathscr{F}\left(f_{1}\right) \times \mathscr{F}\left(f_{2}\right) \\
\mathscr{F}(f, g)=(\mathscr{F}(f), \mathscr{F}(g))
\end{gathered}
$$

for all mappings $f_{1}: M_{1} \rightarrow N_{1}, f_{2}: M_{2} \rightarrow N_{2}, f: M \rightarrow N_{1}$ and $g: M \rightarrow N_{2}$.

From the definition we obtain that a product preserving functor $\mathscr{F}$ has a point-property i.e. $\mathscr{F}$ (point) $=$ point. This implies that for a constant mapping $\varphi: M$ $\rightarrow N$ the induced mapping $\mathscr{F}(\varphi)$ is also constant.

The tangent bundle $T M$ and the tangent bundle of $p^{r}$-velocities $T_{p}^{r} M=$ $J_{0}^{r}\left(\mathbf{R}^{p}, M\right)$ (see [19], [21]) are important examples of product preserving functors. The most general example of product preserving functor is so-called Weil functor (see [24]). It is constructed as follows:

Let $\mathbf{R}[p]=\mathbf{R}\left[\left[X_{1}, \ldots, X_{p}\right]\right]$ be the algebra of all formal power series of $p$ indeterminates $X_{1}, \ldots, X_{p}$ and let $\mathfrak{m}_{p}$ be the maximal ideal of $\mathbf{R}[p]$ of all formal power series without constant terms. Let $a$ be an ideal of $\mathbf{R}[p]$ such that $\mathbf{R}[p] / \mathfrak{a}$ $<\infty$. The algebra $A=\mathbf{R}[p] / \mathfrak{a}$ has the unique maximal ideal $\mathfrak{m}=\mathfrak{m}_{p} / \mathfrak{a}$.

For this algebra $A$ we construct a product preserving functor $T^{A}$.

Let $\xi_{A}: \mathbf{R}[p] \rightarrow A$ be the natural projection. We denote by $\tau: \mathscr{C}^{\infty}\left(\mathbf{R}^{p}\right) \rightarrow$ $\mathbf{R}[p]$ the formal Taylor expansion at the origin $t=0$, i.e. for $f \in \mathscr{C}^{\infty}\left(\mathbf{R}^{p}\right)$ we have

$$
\tau(f)=\sum_{\nu} \frac{1}{\nu !}\left[\left(\frac{\partial}{\partial t}\right)^{\nu} f\right]_{t=0} X^{\nu}
$$

Now we define an equivalence relation in the set $\mathscr{C}^{\infty}\left(\mathbf{R}^{p}, M\right)$ of smooth mappings $\mathbf{R}^{p} \rightarrow M$ (similar to the relation of jets): $\gamma, \gamma^{\prime}: \mathbf{R}^{p} \rightarrow M$ are $A$-equivalent if

$$
\xi_{A}(\tau(f \circ \gamma))=\xi_{A}\left(\tau\left(f \circ \gamma^{\prime}\right)\right)
$$

for every $f \in \mathscr{C}^{\infty}(M)$. We denote by $j^{A} \gamma$ the equivalence class of $\gamma: \mathbf{R}^{p} \rightarrow M$, by $T^{A} M$ the set of all equivalence classes and by $\pi_{A}: T^{A} M \rightarrow M$ the natural projection $\pi_{A}\left(j^{A} \varphi\right)=\varphi(0)$.

For a smooth mapping $\varphi: M \rightarrow N$ we define $T^{A} \varphi: T^{A} M \rightarrow T^{A} N$ by $T^{A} \varphi\left(j^{A} \gamma\right)=j^{A}(\varphi \circ \gamma)$. If $(U, \varphi)$ is a chart on $M$, then $\left(T^{A} U, T^{A} \varphi\right)$ is a chart on $T^{A} M$. It is easy to observe that $T^{A}$ is a product preserving functor.

In 1986 Eck [3], Kainz, Michor [11] and Luciano [16] have proved independently that any product preserving functor is in fact equivalent to some Weil functor. 
THEOREM 1.3. If $\mathscr{F}$ is a product preserving functor, then there exists an algebra $A=\mathbf{R}[p] /$ a such that $\mathscr{F}(M)=T^{A}(M)$ for every manifold $M$.

In the paper we will not use the above theorem.

Product preserving functors have many interesting properties. In this section we formulate some properties of product preserving functors used in the paper. We start from the following proposition:

Proposition 1.4. If $\mathscr{F}$ is a product preserving functor then $A=\mathscr{F}(\mathbf{R})$ is a real associative, commutative and finite dimensional algebra.

If,$+ \because \mathbf{R}^{2} \rightarrow \mathbf{R}$ are the addition and the multiplication on $\mathbf{R}$ and $m_{\alpha}: \mathbf{R} \rightarrow \mathbf{R}$ is the multiplication by $\alpha \in \mathbf{R}$, then $\mathscr{F}(+), \mathscr{F}(\cdot), \mathscr{F}\left(m_{\alpha}\right)$ are the operations in $A, \mathscr{F}(0)$ and $\mathscr{F}(1)$ are the zero and the unity in $A$ (after identification of constant mappings with their values).

The set $N=\mathscr{F}_{0}(\mathbf{R})$ is the ideal of nilpotent elements of $A$. We have $A=\mathbf{R} \cdot 1 \oplus N$.

The algebra $A=\mathscr{F}(\mathbf{R})$ will be called Weil algebra of $\mathscr{F}$.

Proof. $A=\mathscr{F}(\mathbf{R})$ is an algebra by the functoriality of $\mathscr{F}$. For instance, to show the associativity of $\mathscr{F}(+)$ we apply $\mathscr{F}$ to the formula $+\circ(+\times$ id $)=$ $+\circ($ id $\times+)$.

To prove the properties of $N$ we observe that the restriction of $\mathscr{F}$ to the category of 1-dimensional manifolds is a natural bundle, and by [26] it is of finite order $h$. Let $q(t)=t+t^{h+1}$. Since $j_{0}^{h} q=j_{0}^{h} \mathrm{id}$, thus, for $a \in N$ we have

$$
a+a^{h+1}=\mathscr{F}_{0}(q)(a)=a .
$$

It implies $a^{h+1}=0$.

If we apply a product preserving functor to some particular manifolds as groups, vector spaces, vector bundles and so on we obtain many interesting properties. We collect these properties in a few propositions. At the beginning we formulate properties for vector spaces and vector bundles.

PROPOSITION 1.5. Let $\mathscr{F}$ be a product preserving functor.

1. If $V$ is a finite dimensional vector space, then $\mathscr{F}(V)$ is a finite dimensional vector space. If $+: V \times V \rightarrow V$ is the sum mapping in $V$ and for $\alpha \in \mathbf{R}, d_{\alpha}: V \rightarrow V$ is the multiplication by scalar $\alpha$, then $\mathscr{F}(+): \mathscr{F}(V) \times \mathscr{F}(V) \rightarrow \mathscr{F}(V)$ is the sum mapping in $\mathscr{F}(V)$ and $\mathscr{F}\left(d_{\alpha}\right): \mathscr{F}(V) \rightarrow \mathscr{F}(V)$ is the multiplication by scalar $\alpha$ in 
$\mathscr{F}(V)$. The zero of $\mathscr{F}(V)$ is $\mathscr{F}(0)$, where $0: V \rightarrow V$ is the constant zero mapping.

2. If $f: V \rightarrow W$ is a linear mapping of two finite dimensional vector spaces, then $\mathscr{F}(f): \mathscr{F}(V) \rightarrow \mathscr{F}(W)$ is also linear.

3. If $f: V_{1} \times \cdots \times V_{k} \rightarrow W$ is a k-linear mapping, then $\mathscr{F}(f): \mathscr{F}\left(V_{1}\right) \times \cdots$ $\times \mathscr{F}\left(V_{k}\right) \rightarrow \mathscr{F}(W)$ is also $k$-linear.

4. If $V=U_{1} \oplus U_{2}$ is a direct sum of subspaces $U_{1}, U_{2}$, then we have $\mathscr{F}(V)=$ $\mathscr{F}\left(U_{1}\right) \oplus \mathscr{F}\left(U_{2}\right)$.

5. If $f: V \rightarrow W$ is linear, then we have

$$
\operatorname{ker} \mathscr{F}(f)=\mathscr{F}(\operatorname{ker} f), \quad \operatorname{im} \mathscr{F}(f)=\mathscr{F}(\operatorname{im} f) .
$$

Proof. The proofs of parts 1-4 are standard (cf. the proof of Proposition 1.4). To show part 5 we consider a subspace $U$ such that $V=\operatorname{ker} f \oplus U$. We denote by $i: \operatorname{ker} f \rightarrow V$ and $j: U \rightarrow V$ the inclusions. If we apply $\mathscr{F}$ to the equality $f \circ i=0$ and to the isomorphism $f \circ j: U \rightarrow \operatorname{im} f$ we obtain $\mathscr{F}(f)_{\mid \mathscr{F}(\text { ker } f)}=0$ and the isomorphism $\mathscr{F}(f)_{\mid \mathscr{F}(U)}: \mathscr{F}(U) \rightarrow \mathscr{F}(\operatorname{im} f)$. This implies (1.3).

For a vector space $V$ we can define a richer structure on $\mathscr{F}(V)$. Namely, we have

Proposition 1.6. Let $\mathscr{F}$ be a product preserving functor and let $A=\mathscr{F}(\mathbf{R})$ be its Weil algebra.

1. If $V$ is a finite dimensional vector space, then $\mathscr{F}(V)$ is an $A$-module. If $m: \mathbf{R}$ $\times V \rightarrow V$ is the scalar multiplication in $V$, then the induced mapping $\mathscr{F}(m): A \times$ $\mathscr{F}(V) \rightarrow \mathscr{F}(V)$ defines the action of $A$ on $\mathscr{F}(V)$.

2. If $f: V \rightarrow W$ is a linear mapping, then $\mathscr{F}(f): \mathscr{F}(f) \rightarrow \mathscr{F}(W)$ is a homomorphism of $A$-modules. If $f: V_{1} \times \cdots \times V_{k} \rightarrow W$ is $k$-linear over $\mathbf{R}$, then $\mathscr{F}(f)$ : $\mathscr{F}\left(V_{1}\right) \times \cdots \times \mathscr{F}\left(V_{k}\right) \rightarrow \mathscr{F}(W)$ is $k$-linear over $A$.

3. If $v_{1}, \ldots, v_{n}$ is a basis of a vector space $V$, then $\mathscr{F}\left(v_{1}\right), \ldots, \mathscr{F}\left(v_{n}\right)$ is a basis of the $A$-module $\mathscr{F}(V)$. Furthermore, if $a_{1}, \ldots, a_{K}$ is a basis of $A$ over $\mathbf{R}$, then all products $a_{\nu} \mathscr{F}\left(v_{i}\right)$, where $i=1, \ldots, n$ and $\nu=1, \ldots, K$, is a basis of $\mathscr{F}(V)$ over $\mathbf{R}$.

The proof of parts 1 and 2 is standard. To show part 3 we apply $\mathscr{F}$ to the isomorphism $\mathbf{R}^{n} \ni\left(\xi^{1}, \ldots, \xi^{n}\right) \rightarrow \sum_{i=1}^{n} \xi^{i} v_{i} \in V$.

We can prolong these properties to category of vector bundles. In this case we obtain 
PRoposition 1.7. Let $\mathscr{F}$ be a product preserving functor.

1. If $\pi: E \rightarrow M$ is a vector bundle, then $\mathscr{F}(\pi): \mathscr{F}(E) \rightarrow \mathscr{F}(M)$ is a vector bundle too. If $V$ is the standard fibre of $E$ and $\varphi: E_{\mid U} \rightarrow U \times V$ is a trivialization over an open subset $U \subset M$, then $\mathscr{F}(V)$ is the standard fibre of $\mathscr{F}(E)$ and $\mathscr{F}(\varphi)$ : $\mathscr{F}(E)_{\mid \mathscr{F}(U)} \rightarrow \mathscr{F}(U) \times \mathscr{F}(V)$ is a trivialization over $\mathscr{F}(U) \subset \mathscr{F}(M)$.

2. If $\Psi: E \rightarrow E^{\prime}$ is a vector bundle homomorphism, then $\mathscr{F}(\Psi): \mathscr{F}(E) \rightarrow$ $\mathscr{F}\left(E^{\prime}\right)$ is also a vector bundle homomorphism.

3. Let $E_{1}, \ldots, E_{k}$ be vector bundle over the same base $M$ and $E$ be a vector bundle over $N$. If $\Psi: E_{1} \times{ }_{M} \cdots \times{ }_{M} E_{k} \rightarrow E$ is a $k$-linear mapping covering $\phi: M \rightarrow N,{ }^{1)}$ then $\mathscr{F}(\Psi): \mathscr{F}\left(E_{1}\right) \times_{\mathscr{F}(M)} \cdots \times_{\mathscr{F}(M)} \mathscr{F}\left(E_{k}\right) \rightarrow \mathscr{F}(E)$ is a $k$-linear mapping covering $\mathscr{F}(\phi): \mathscr{F}(M) \rightarrow \mathscr{F}(N)$.

4. If $E=E_{1} \oplus E_{2}$ is a direct sum of two vector bundles $E_{1}$ and $E_{2}$, then $\mathscr{F}(E)$ : $\mathscr{F}\left(E_{1}\right) \oplus \mathscr{F}\left(E_{2}\right)$.

5. If $f: E \rightarrow E^{\prime}$ is a vector bundle homomorphism such that the function $x \rightarrow$ $\operatorname{dim} \operatorname{ker} f_{x}$ is constant on the base of $E^{2)}$, then

$$
\operatorname{ker} \mathscr{F}(f)=\mathscr{F}(\operatorname{ker} f), \quad \operatorname{im} \mathscr{F}(f)=\mathscr{F}(\operatorname{im} f) .
$$

For Lie groups and their actions on manifolds we collect properties interesting for us in the followlng proposition.

Proposition 1.8. Let $\mathscr{F}$ be a product preserving functor and $A=\mathscr{F}(\mathbf{R})$ be its Weil algebra.

1. If $G$ is a Lie group, then $\mathscr{F}(G)$ is also a Lie group. If $m: G \times G \rightarrow G$ is the product in $G$ and 1 is the unity of $G$, then $\mathscr{F}(m)$ is the product in $\mathscr{F}(G)$ and $\mathscr{F}(1)$ is the unity of $\mathscr{F}(G)$.

2. If a Lie group $G$ acts on a manifold $M$ and $\Lambda: G \times M \rightarrow M$ is the action, then $\mathscr{F}(G)$ acts on $\mathscr{F}(M)$ and $\mathscr{F}(\Lambda): \mathscr{F}(G) \times \mathscr{F}(M) \rightarrow \mathscr{F}(M)$ is the action.

In particular, if ad: $G \times G \rightarrow G$ is the adjoint action of $G$ on $G$, then $\mathscr{F}(\mathrm{ad})$ : $\mathscr{F}(G) \times \mathscr{F}(G) \rightarrow \mathscr{F}(G)$ is the adjoint action of $\mathscr{F}(G)$ on $\mathscr{F}(G)$.

If $\rho: G L\left(\mathbf{R}^{n}\right) \times \mathbf{R}^{n} \rightarrow \mathbf{R}^{n}$ is the standard action, then $\mathscr{F}(\rho)$ gives an action on $\mathscr{F}\left(\mathbf{R}^{n}\right)$ of $A$-linear transformations and we have a Lie group monomorphism $I: \mathscr{F}\left(G L\left(\mathbf{R}^{n}\right)\right) \rightarrow G L_{A}\left(\mathscr{F}\left(\mathbf{R}^{n}\right)\right) \subset G L\left(\mathscr{F} \mathbf{R}^{n}\right)$ given by $I(X)(y)=\mathscr{F}(\rho)(X, y)$ for $X \in \mathscr{F}\left(G L\left(\mathbf{R}^{n}\right)\right)$ and $y \in \mathscr{F}\left(\mathbf{R}^{n}\right)$.

3. If $f: G \rightarrow G^{\prime}$ is a Lie group homomorphism, then $\mathscr{F}(f): \mathscr{F}(G) \rightarrow \mathscr{F}\left(G^{\prime}\right)$ is a

\footnotetext{
1) It means that for each point $x \in M \Psi$ transfoms $\left(E_{1}\right)_{x} \times \cdots \times\left(E_{k}\right)_{x}$ into $E_{\psi(x)}$ and $\Psi_{x}=\Psi_{\mid\left(E_{1}\right)_{x} \times \cdots \times\left(E_{k}\right)_{x}}:\left(E_{1}\right)_{x} \times \cdots \times\left(E_{k}\right)_{x} \rightarrow E_{\psi(x)}$ is $k$-linear.

2) This assumption gives a sufficient and necessary condition under which $\operatorname{im} f \subset E^{\prime}$ and Ker $f \subset E$ are vector subbundles.
} 
Lie group homomorphism. Particularly, if $H \subset G$ is a Lie subgroup, then $\mathscr{F}(H)$ is a Lie subgroup of $\mathscr{F}(G)$.

4. If $P(M, G, \pi)$ is a principal fibre bundle with base $M$, structure group $G$ and projection $\pi$, then $\mathscr{F}(P)(\mathscr{F}(M), \mathscr{F}(G), \mathscr{F}(\pi))$ is a principal fibre bundle with base $\mathscr{F}(M)$, structure group $\mathscr{F}(G)$ and projection $\mathscr{F}(\pi)$. If $\varphi: P_{\mid U} \rightarrow U \times G$ is a trivialization over $U$, then $\mathscr{F}(\varphi): \mathscr{F}(P)_{\mid \mathscr{F}(U)} \rightarrow \mathscr{F}(U) \times \mathscr{F}(G)$ is a trivialization over $\mathscr{F}(U)$.

5. If $f: P(M, G) \rightarrow P^{\prime}\left(M^{\prime}, G^{\prime}\right)$ is a homomorphism of principal fibre bundles covering $\varphi: M \rightarrow M^{\prime}$ with an induced Lie group homomorphism $\rho_{f}: G \rightarrow G^{\prime}$, then $\mathscr{F}(f): \mathscr{F}(P) \rightarrow \mathscr{F}\left(P^{\prime}\right)$ is homomorphism of principal fibre bundles covering $\mathscr{F}(\varphi)$ : $\mathscr{F}(M) \rightarrow \mathscr{F}\left(M^{\prime}\right)$ and $\mathscr{F}\left(\rho_{f}\right): \mathscr{F}(G) \rightarrow \mathscr{F}\left(G^{\prime}\right)$ is the induced lie group homomorph ism.

Proof. The unique nonstandard step of the proof is the injectivity of $I$ from part 2.

Applying $\mathscr{F}$ to $\mathbf{R}^{n^{2}} \times \mathbf{R}^{n} \supset G L\left(\mathbf{R}^{n}\right) \times \mathbf{R}^{n} \stackrel{\rho}{\rightarrow} \mathbf{R}^{n}$ we obtain

$$
A^{n^{2}} \times A^{n^{2}}=\mathscr{F}\left(\mathbf{R}^{n^{2}}\right) \times \mathscr{F}\left(\mathbf{R}^{n^{2}}\right) \supset \mathscr{F}\left(G L\left(\mathbf{R}^{n}\right)\right) \times \mathscr{F}\left(\mathbf{R}^{n}\right) \stackrel{\mathscr{F}(\rho)}{\longrightarrow} \mathscr{F}\left(\mathbf{R}^{n}\right)
$$

given by $\mathscr{F}(\rho)\left(\left[x_{j}^{i}\right],\left(x^{k}\right)\right)=\left(\sum_{j=1}^{n} x_{j}^{i} x^{j}\right)$. This formula implies the injectivity of I.

There is a very interesting and nontrivial property saying that for two product preserving functors $\mathscr{F}_{1}, \mathscr{F}_{2}$ there exists a natural isomorphism $\mathscr{F}_{1}\left(\mathscr{F}_{2}(M)\right) \rightarrow$ $\mathscr{F}_{2}\left(\mathscr{F}_{1}(M)\right)$. We will use this property only in the case when one of these functors is the tangent bundle. We have

Proposition 1.9. Let $\mathscr{F}$ be a product preserving functor. There exists one and only one family $\eta_{M}: \mathscr{F}(T M) \rightarrow T \mathscr{F}(M)$ of vector bundle isomorphisms

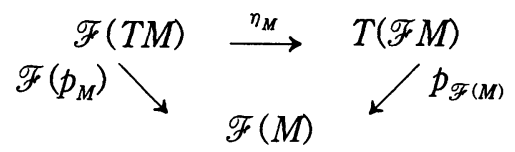

(where $p_{M}: T M \rightarrow M$ is the projection) such that the following conditions hold:

(1) for every smooth mapping $\varphi: M \rightarrow N$ the following diagram 


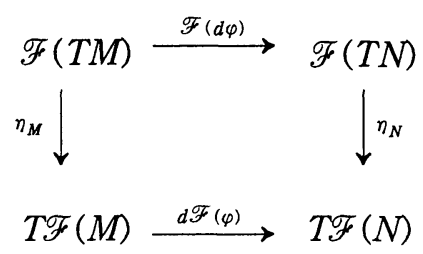

commutes;

(2) for two manifolds $M, N$ we have $\eta_{M \times N}=\eta_{M} \times \eta_{N}$;

(3) if $\Psi_{\mathbf{R}}: T \mathbf{R} \rightarrow \mathbf{R} \times \mathbf{R}$ and $\Psi_{\mathscr{F}(\mathbf{R})}: T \mathscr{F}(\mathbf{R}) \rightarrow \mathscr{F}(\mathbf{R}) \times \mathscr{F}(\mathbf{R})$ are the standard trivializations, then

$$
\eta_{\mathbf{R}}: \mathscr{F}(T \mathbf{R}) \stackrel{\mathscr{F}\left(\Psi_{\mathbf{R}}\right)}{\longrightarrow} \mathscr{F}(\mathbf{R} \times \mathbf{R})=\mathscr{F}(\mathbf{R}) \times \mathscr{F}(\mathbf{R}) \stackrel{\Psi_{\mathscr{F}}^{-1}(\mathbf{R})}{\longrightarrow} T(\mathscr{F}(\mathbf{R})) .
$$

Outline of the proof. Let us observe that according to (2) we need to define $\eta_{\mathbf{R}^{n}}=\left(\eta_{\mathbf{R}}\right)^{n}$. Now (1) holds for $M=\boldsymbol{R}^{n}$ and $N=\mathbf{R}^{m}$. Next using charts we can define $\eta_{M}$ for any $M$ such that (1) holds.

In [9] we have proved that condition (2) is a consequence of condition (1) from Proposition 1.9.

We finish this section by remarks on Lie algebras. We have

PROPOSITION 1.10. Let $\mathscr{F}$ be a product preserving functor.

1. If $\mathfrak{g}$ is a Lie algebra, then $\mathscr{F}(\mathfrak{g})$ is also a Lie algebra with the Lie bracket $\mathscr{F}([]$,$) , where []:, \mathrm{g} \times \mathrm{g} \rightarrow \mathrm{g}$ is the Lie bracket in $\mathrm{g}$.

2. If $f: \mathfrak{g} \rightarrow \mathfrak{g}^{\prime}$ is a Lie algebra homomorphism, then $\mathscr{F}(f): \mathscr{F}(\mathfrak{g}) \rightarrow \mathscr{F}\left(g^{\prime}\right)$ is also a Lie algebra homomorphism.

3. If $G$ is a Lie group and $\mathscr{L}(G)$ is its Lie algebra, then the restriction

$$
\left(\eta_{G}\right)_{\mid \mathscr{F}(\mathscr{L}(G))}: \mathscr{F}(\mathscr{L}(G)) \rightarrow \mathscr{L}(\mathscr{F}(G))
$$

is a Lie algebra isomorphism, where $\eta_{G}$ is from Proposition 1.9. The restriction $\left(\eta_{G}\right)_{\mid \mathscr{F}(\mathscr{L}(G))}$ will be denoted also by $\eta_{G}$.

The verifications of parts 1 and 2 are standard. We will prove part 3 in Section 2. 


\section{Lifts of functions and vector fields}

Let $\mathscr{F}$ be a product preserving functor and let $A=\mathscr{F}(\mathbf{R})$ be the corresponding Weil algebra.

If $\lambda: A \rightarrow \mathbf{R}$ is a smooth function and $f: M \rightarrow \mathbf{R}$ is a smooth function on a manifold $M$, then we define $\lambda$-lift of $f$ to $\mathscr{F}(M)$

$$
f^{(\lambda)}=\lambda \circ \mathscr{F}(f) .
$$

$f^{(\lambda)}$ is a smooth function on $\mathscr{F}(M)$. It is easy to verify

PROPOSITION 2.1. Let $\mathscr{F}$ be a product preserving functor and $A=\mathscr{F}(\mathbf{R})$ be its Weil algebra. For any smooth function $\lambda: A \rightarrow \mathbf{R}$ the family of mappings $\mathscr{C}^{\infty}(M) \ni$ $f \rightarrow f^{(\lambda)} \in \mathscr{C}^{\infty}(\mathscr{F} M)$ is a lifting, i.e. for every smooth mapping $\varphi: M \rightarrow N$ and every function $f \in \mathscr{C}^{\infty}(N)$ we have $(f \circ \varphi)^{(\lambda)}=f^{(\lambda)} \circ \mathscr{F}(\varphi)$.

The $\lambda$-lift have the following properties, being an immediate consequence of the definition.

If $\lambda_{1}, \lambda_{2}: A \rightarrow \mathbf{R}$ are two smooth functions and $\alpha_{1}, \alpha_{2}$ are reals, then

$$
f^{\left(\alpha_{1} \lambda_{1}+\alpha_{2} \lambda_{2}\right)}=\alpha_{1} f^{\left(\lambda_{1}\right)}+\alpha_{2} f^{\left(\lambda_{2}\right)} .
$$

If $\lambda: A \rightarrow \mathbf{R}$ is linear and $\alpha, \beta$ are reals, then

$$
(\alpha f+\beta g)^{(\lambda)}=\alpha f^{(\lambda)}+\beta g^{(\lambda)} .
$$

Let $\mathscr{B}=\left(a_{1}, \ldots, \alpha_{K}\right)$ be a basis of $A$ over $\mathbf{R}$. We consider $\mathscr{B}$ as a linear isomorphism $A \rightarrow \mathbf{R}^{K}$ and let $\pi_{\mathscr{B}}^{\nu}: A \rightarrow \mathbf{R}$ be the composition of $\mathscr{B}$ with the projection $\mathbf{R}^{K} \rightarrow \mathbf{R}$ on $\nu$-th factor, $\nu=1, \ldots, K$.

For a coordinate system $\left(U, x^{1}, \ldots, x^{n}\right)$ in $M$ we define the induced coordinate system $\left\{x^{i, \nu}\right\}$ on $\mathscr{F}(M)$ by

$$
x^{i, \nu}=\left(x^{i}\right)^{\left(\pi_{\circledast}^{\nu}\right)}, \text { for } \nu=1, \ldots, K .
$$

From (2.4) we obtain immediately

Proposition 2.2. Let $\mathscr{F}$ be a product preserving functor and $A=\mathscr{F}(\mathbf{R})$ be its Weil algebra.

If $\tilde{X}, \tilde{Y}$ are vector fields on $\mathscr{F}(M)$ such that for every smooth function $f$ on $M$ and every linear function $\lambda: A \rightarrow \mathbf{R}$ we have $\tilde{X}\left(f^{(\lambda)}\right)=\tilde{Y}\left(f^{(\lambda)}\right)$, then $\tilde{X}=\tilde{Y}$. 
Next we will study lifts of vector fields. The standard example is so-called complete lift. If $X: M \rightarrow T M$ is a vector field on $M$, then we define $X^{C}=\eta_{M}$ 。 $\mathscr{F}(X)$, where $\eta_{M}$ is the isomorphism from Proposition 1.9. It is a vector field on $\mathscr{F}(M)$ called complete lift of $X$ to $\mathscr{F}(M)$. We have

Proposition 2.3. Let $\mathscr{F}$ be a product preserving functor and $A=\mathscr{F}(\mathbf{R})$ be its Weil algebra. If $\varphi_{t}$ is a local flow of $X$, then $\mathscr{F}\left(\varphi_{t}\right)$ is a local flow of $X^{C}$.

Proof. It is clear that $\mathscr{F}\left(\varphi_{t}\right)$ is a local flow on $\mathscr{F}(M)$. Let $\tilde{X}$ be the local vector field on $\mathscr{F}(M)$ defined by $\mathscr{F}\left(\varphi_{t}\right)$.

According to the commutativity of the condition (1) from Proposition 1.9 we can assume that $M=\mathbf{R}^{n}$.

For a point $y \in \mathscr{F}\left(\mathbf{R}^{n}\right)$ the coordinates of $\tilde{X}(y)$ are equal to $\left.\frac{d}{d t} \mathscr{F}\left(\varphi_{t}\right)(y)\right|_{t=0}$ $\in A^{n}=\mathscr{F}\left(\mathbf{R}^{n}\right)$. On the other hand the coordinates of $\left(\eta_{\mathbf{R}^{n}} \circ \mathscr{F}(X)\right)(y)$ are equal to $F\left(\left.\frac{d}{d t} \varphi_{t}\right|_{t=0}\right)(y)=\left.\frac{d}{d t} \mathscr{F}\left(\varphi_{t}\right)(y)\right|_{t=0}$. In the last equality we apply that for any vector space $V$ the mapping

$$
\mathscr{C}^{\infty}(M, V) \ni h \rightarrow \mathscr{F}(h) \in \mathscr{C}^{\infty}(\mathscr{F}(M), \mathscr{F}(V))
$$

is linear.

From the above proposition we obtain that the complete lift introduced here coincides with the standard definition by flows which is introduced on natural bundles. The complete lift has the following properties (see Salvioli [27], cf. also [5])

$$
(\alpha X+\beta Y)^{C}=\alpha X^{C}+\beta Y^{C}, \quad\left([X, Y]^{C}=\left[X^{C}, Y^{C}\right]\right.
$$

for all vector fields $X, Y$ on $M$ and all reals $\alpha, \beta$.

We will define other examples of lifts of vector fields to $\mathscr{F}$. We consider the mapping $\Psi: \mathbf{R} \times T M \rightarrow T M$ given by $\Psi(t, v)=t v$. Using the natural isomorphisms $\eta_{M}: \mathscr{F}(T M) \rightarrow T(\mathscr{F} M)$, the induced mapping $\mathscr{F}(\Psi): A \times \mathscr{F}(T N) \rightarrow \mathscr{F}(T M)$ determines the mapping

$$
\tilde{\Psi}=\eta_{M} \circ \mathscr{F}(\Psi) \circ\left(\mathrm{id}_{A} \times \eta_{M}^{-1}\right): A \times T(\mathscr{F} M) \rightarrow T(\mathscr{F} M) .
$$

For an element $a \in A$ and a vector $\tilde{v} \in T(\mathscr{F} M)$ we define

$$
a \cdot \tilde{v}=\tilde{\Psi}(a, \tilde{v})
$$


This product has the following properties

$$
\begin{aligned}
(a+b) \cdot \tilde{v} & =a \cdot \tilde{v}+b \cdot \tilde{v}, \\
(a b) \cdot \tilde{v} & =a \cdot(b \cdot \tilde{v}), \\
a \cdot(\tilde{v}+\tilde{w}) & =a \cdot \tilde{v}+a \cdot \tilde{w}, \\
d \mathscr{F}(\varphi)(a \cdot \tilde{v}) & =a \cdot d \mathscr{F}(\varphi)(\tilde{v})
\end{aligned}
$$

for all $a, b \in A, \tilde{v}, \tilde{w} \in T_{y} \mathscr{F}(M), y \in \mathscr{F}(M)$ and every smooth mapping $\varphi: M \rightarrow$ $N$. These properties are a simple consequence of the definition of “.". We verify only the last formula from (2.8). A verification of rest formulas are similar.

The equality $d \varphi(t v)=t d \varphi(v)$ can be written without arguments in the form $d \varphi \circ \Psi=\Psi \circ\left(\operatorname{id}_{\mathbf{R}} \times d \varphi\right)$. Applying $\mathscr{F}$ and composing the obtained formula with $\eta_{N}$ and $\mathrm{id}_{A} \times \eta_{M}^{-1}$ we obtain

$$
\eta_{N} \circ \mathscr{F}(d \varphi) \circ \mathscr{F}(\Psi) \circ\left(\mathrm{id}_{A} \times \eta_{M}^{-1}\right)=\eta_{N} \circ \mathscr{F}(\Psi) \circ\left(\mathrm{id}_{A} \times \mathscr{F}(d \varphi) \circ \eta_{M}^{-1}\right) .
$$

Using the commutativity of the second diagram from Proposition 1.9 we deduce

$$
\begin{aligned}
d \mathscr{F}(\varphi) \circ \tilde{\Psi} & =d \mathscr{F}(\varphi) \circ \eta_{M} \circ \mathscr{F}(\Psi) \circ\left(\mathrm{id}_{A} \times \eta_{M}^{-1}\right) \\
& =\eta_{N} \circ \mathscr{F}(d \varphi) \circ \mathscr{F}(\Psi) \circ\left(\mathrm{id}_{A} \times \eta_{M}^{-1}\right) \\
& =\eta_{N} \circ \mathscr{F}(\Psi) \circ\left(\mathrm{id}_{A} \times \mathscr{F}(d \varphi) \circ \eta_{M}^{-1}\right) \\
& =\bar{\Psi} \circ\left(\mathrm{id}_{A} \times \eta_{N} \circ \mathscr{F}(d \varphi) \circ \eta_{M}^{-1}\right) \\
& =\bar{\Psi} \circ\left(\mathrm{id}_{A} \times d \mathscr{F}(\varphi)\right) .
\end{aligned}
$$

By the definition of "." the above equality is equivalent to the last formula from (2.8).

Let us also observe that after identification $T_{y}\left(\mathscr{F}\left(\mathbf{R}^{n}\right)\right)$ with $\mathscr{F}\left(\mathbf{R}^{n}\right)$ we have

$$
a \cdot \tilde{v}=a \tilde{v}
$$

for $a \in A$ and $\tilde{v} \in T_{y}\left(\mathscr{F}\left(\mathbf{R}^{n}\right)\right)=\mathscr{F}\left(\mathbf{R}^{n}\right), y \in \mathscr{F}\left(\mathbf{R}^{n}\right)$, where $A$-module structure on $\mathscr{F}\left(\mathbf{R}^{n}\right)$ is defined in Proposition 1.6.

Now for a vector field $X$ on $M$ and an element $a \in A$ we define

$$
X^{(a)}=a \cdot X^{C}=\tilde{\Psi}\left(a, X^{C}\right) .
$$

$X^{(a)}$ is a vector field on $\mathscr{F}(M)$ called $a$-lift of $X$. This $a$-lift was introduced by Koláŕ [14]. From (2.9) we obtain immediately

$$
X^{C}=X^{(1)} \text {, }
$$

where 1 is the unit of $A$. Kolár̆ in [13] proved 
Proposition 2.4. Let $\mathscr{F}$ be a product preserving functor, $A=\mathscr{F}(\mathbf{R})$ be its Weil algebra and $a \in A$. The family of mappings $\mathscr{X}(M) \ni X \rightarrow X^{(a)} \in \mathscr{X}(\mathscr{F} M)$ is a lifting i.e. for every smooth mapping $\varphi: M \rightarrow N$ and every $\varphi$-related vector fields $X \in$ $\mathscr{X}(M), Y \in \mathscr{X}(N)$ the vector fields $X^{(a)} \in \mathscr{X}(\mathscr{F}(M)), Y^{(a)} \in \mathscr{X}(\mathscr{F}(N))$ are $\mathscr{F}(\varphi)$ related.

Proof. We apply $\mathscr{F}$ to diagram (0.1) and next we use formulas (2.7) and (2.8).

We have the following proposition on flows of $X^{(a)}$.

Proposition 2.5. Let $\mathscr{F}$ be a product preserving functor and $A=\mathscr{F}(\mathbf{R})$ be its Weil algebra. If $\varphi:(-\varepsilon,+\varepsilon) \times U \rightarrow M$ is a local flow of a vector field $X$ on $M$, then the mapping $\tilde{\varphi}:\left(-\varepsilon^{\prime},+\varepsilon^{\prime}\right) \times \mathscr{F}(M) \mid U \rightarrow \mathscr{F}(M)$ given by

$$
\tilde{\varphi}(t, y)=\mathscr{F}(\varphi)(t a, y)
$$

is a local flow of $X^{(a)}$.

Proof. Let us observe that from the equality $\varphi(t+s, x)=\varphi(t, \varphi(s, x))$ the induced mapping $\mathscr{F}(\varphi): A_{\mid(-\varepsilon,+\varepsilon)} \times \mathscr{F}(M)_{\mid U} \rightarrow \mathscr{F}(M)$ satisfies the condition $\mathscr{F}(\varphi)(a+b, y)=\mathscr{F}(\varphi)(a, \mathscr{F}(\varphi)(b, y))$ for all $a, b$ and all $y$ such that $a, b$, $a+b \in A_{\mid(-\varepsilon,+\varepsilon)}$ and $y, \mathscr{F}(\varphi)(b, y) \in \mathscr{F}(M)_{\mid U}$. It implies that $\tilde{\varphi}$ is a local flow on $\mathscr{F}(M)$.

Without lost of the generality we can assume $M=\mathbf{R}^{n}$. Let $\tilde{X}$ be a local vector field on $\mathscr{F}\left(\mathbf{R}^{n}\right)$ defined by $\tilde{\varphi}$. For a point $y \in \mathscr{F}\left(\mathbf{R}^{n}\right)$ by (2.9) and Proposition 2.3 the coordinates of $X^{(a)}$ at $y$ are equal to $\left.a \frac{d}{d t} \mathscr{F}\left(\varphi_{t}\right)(y)\right|_{t=0} \in A^{n}=\mathscr{F}\left(\mathbf{R}^{n}\right)$. The coordinates of $\tilde{X}$ at $y$ are equal to

$$
\begin{aligned}
\frac{d}{d t} \mathscr{F}(\varphi)(t a, y)_{\mid t=0} & =d_{(0, y)} \mathscr{F}(\varphi)(a, 0) \\
& =a \cdot d_{(0, y)} \mathscr{F}(\varphi)(1,0) \quad \text { by formula }(2.8) \\
& =a \frac{d}{d t} \mathscr{F}(\varphi)(t, y)_{\mid t=0} \\
& =a \frac{d}{d t} \mathscr{F}\left(\varphi_{t}\right)(y)_{\mid t=0}
\end{aligned}
$$

because $\varphi_{t}=\varphi^{\circ}\left(t \times \mathrm{id}_{\mathbf{R}^{n}}\right)$, where $t: \mathbf{R} \rightarrow \mathbf{R}$ is the constant mapping. 
We can prove the following properties of $a$-lifts of vector fields.

Proposition 2.6. Let $\mathscr{F}$ be a product preserving functor and $A=\mathscr{F}(\mathbf{R})$ be its Weil algebra.

1. If $X$ is a vector field on $M, f$ is a function on $M, \lambda: A \rightarrow \mathbf{R}$ is a linear function and $a \in A$, then

$$
X^{(a)}\left(f^{(\lambda)}\right)=(X f)^{\left(2 \circ l_{a}\right)},
$$

where $l_{a}: A \rightarrow A$ is given by $l_{a}(b)=a b$.

2. If $X, Y$ are vector fields on $M, \alpha, \beta$ are reals and $a, b \in A$, then

$$
\begin{aligned}
(\alpha X+\beta Y)^{(a)} & =\alpha X^{(a)}+\beta Y^{(a)} \\
X^{(\alpha a+\beta b)} & =\alpha X^{(a)}+\beta X^{(b)} \\
{\left[X^{(a)}, Y^{(b)}\right] } & =[X, Y]^{(a b)} .
\end{aligned}
$$

3. If $X$ is a left invariant vector field on a Lie group $G$ and $a \in A$, then $X^{(a)}$ is a left invariant vector field on $\mathscr{F}(G)$.

Proof. In the proof of formula (2.12) we can assume that $M=\mathbf{R}^{n}$. By Proposition 2.5 , the last formula from (2.8) and the linearity of $\lambda$ we obtain

$$
\begin{aligned}
\left(X^{(a)} f^{(\lambda)}\right)(y) & =\left.\frac{d}{d t}\left(f^{(\lambda)} \circ \tilde{\varphi}_{t}\right)(y)\right|_{t=0} \\
& =\left.\frac{d}{d t}(\lambda \circ \mathscr{F}(f \circ \varphi))(t a, y)\right|_{t=0} \\
& =\lambda\left(\left.\frac{d}{d t}(\mathscr{F}(f \circ \varphi))(t a, y)\right|_{t=0}\right) \\
& =\lambda\left(d_{(0, y)} \mathscr{F}(f \circ \varphi)(a, 0)\right) \\
& =\lambda\left(a \circ d_{(0, y)} \mathscr{F}(f \circ \varphi)(1,0)\right) \\
& =\left(\lambda \circ l_{a}\right)\left(\left.\frac{d}{d t} \mathscr{F}(f \circ \varphi)(t, y)\right|_{t=0}\right) \\
& =\left(\lambda \circ l_{a}\right)\left(\left.\frac{d}{d t} \mathscr{F}\left(f \circ \varphi_{t}\right)(y)\right|_{t=0}\right) \\
& =\left(\lambda \circ l_{a}\right)\left(\mathscr{F}\left(\left.\frac{d}{d t}\left(f \circ \varphi_{t}\right)\right|_{t=0}\right)(y)\right) \\
& =\left(\lambda \circ l_{a} \circ \mathscr{F}(X f)\right)(y) \\
& =(X f)^{\left(\lambda \circ l_{a}\right)}(y) .
\end{aligned}
$$

The proof of formula (2.12) is finished. 
Formulas (2.13) and (2.14) are an immediate consequence of the definition of $a$-lift and (2.6). To show formula (2.15) let us observe that for every function $f$ on $M$ and every linear function $\lambda: A \rightarrow \mathbf{R}$ by (2.12) and the formula $l_{a}{ }^{\circ} l_{b}=l_{a b}$ we have

$$
\begin{aligned}
{\left[X^{(a)}, Y^{(b)}\right]\left(f^{(\lambda)}\right) } & =X^{(a)}\left(Y^{(b)}\left(f^{(\lambda)}\right)\right)-Y^{(b)}\left(X^{(a)}\left(f^{(\lambda)}\right)\right) \\
& =(X(Y(f)))^{\left(\lambda \circ l_{a} o_{b}\right)}-(Y(X(f)))^{\left(\lambda_{0} l_{b} \circ l_{a}\right)} \\
& =([X, Y](f))^{\left(\lambda \circ l_{a b}\right)} \\
& =[X, Y]^{(a b)}\left(f^{(\lambda)}\right) .
\end{aligned}
$$

Now using Proposition 2.2 we obtain (2.15).

To prove the part 3 we observe that for a flow $\varphi$ of $X$ we have $\varphi(t, \xi g)=$ $\xi \varphi(t, g)$ for $t \in \mathbf{R}, \xi, g \in G$. Applying $\mathscr{F}$ we deduce $\mathscr{F}(\varphi)(b, \tilde{\xi} \tilde{g})=$ $\tilde{\xi} \mathscr{F}(\varphi)(b, \tilde{g})$ for all $b \in A, \tilde{\xi}, \tilde{g} \in \mathscr{F}(G)$. In particular $\mathscr{F}(\varphi)(t a, \tilde{\xi} \tilde{g})=$ $\tilde{\xi} \mathscr{F}(\varphi)(t a, \tilde{g})$. Since $\mathscr{F}(\varphi)(t a, y)$ is a flow of $X^{(a)}$, thus $X^{(a)}$ is left invariant.

Formula (2.12) is very useful in the calculations (for instance it is used in the proof of (2.15)). In future we will use also a similar formula for vector-valued functions.

Proposition 2.7. Let $\mathscr{F}$ be a product preserving functor and $A=\mathscr{F}(\mathbf{R})$ be its Weil algebra. Let $V$ be a finite dimensional vector space. For any vector field $X$ on $M$, any $a \in A$ and any smooth function $f: M \rightarrow V$ we have

$$
X^{(a)}(\mathscr{F}(f))=a \mathscr{F}(X f) .
$$

The proof is similar to the verification of formula (2.12).

From (2.4) and (2.12) we deduce

Corollary 2.8. Let $\left(U, x^{i}\right)$ be a chart on $M, \partial_{1}, \ldots, \partial_{n}$ be its adapted frame and let $a_{1}, \ldots, a_{K}$ be a basis of $A$. Then $\left\{\left(\partial_{i}\right)^{\left(a_{\nu}\right)} \mid i=1, \ldots, n, \nu=1, \ldots, K\right\}$ is the adapted frame to the induced chart $\left(\mathscr{F}(U), x^{i, \nu}\right)$ on $\mathscr{F}(M)$.

From Corollary 2.8 we obtain immediately

Proposition 2.9. Let $\mathscr{F}$ be a product preserving functor and $A=\mathscr{F}(\mathbf{R})$ be its Weil algebra. If $\tilde{S}, \tilde{S}^{\prime}$ are two tensor fields of type $(1, k)$ or $(0, k)$ on $\mathscr{F}(M)$ such that for all vector fields $X_{1}, \ldots, X_{k}$ on $M$ and all elements $a_{1}, \ldots, a_{k}$ of $A$ the equality 


$$
\tilde{S}\left(X_{1}^{\left(a_{1}\right)}, \ldots, X_{k}^{\left(a_{k}\right)}\right)=\tilde{S}^{\prime}\left(X_{1}^{\left(a_{1}\right)}, \ldots, X_{k}^{\left(a_{k}\right)}\right)
$$

holds, then $\tilde{S}=\tilde{S}^{\prime}$.

Now we can prove the following proposition formulated in Section 1 (see Proposition $1.10(3))$.

Proposition 2.10. Let $\mathscr{F}$ be a product preserving functor. If $G$ is a Lie group and $\mathscr{L}(G)$ is its Lie algebra, then the restriction $\left(\eta_{G}\right)_{\mid \mathscr{F}(\mathscr{L}(G))}: \mathscr{F}(\mathscr{L}(G)) \rightarrow \mathscr{L}(\mathscr{F}(G))$ is a Lie algebra isomorphism, where $\eta_{G}$ is from Proposition 1.9.

The restriction $\left(\eta_{G}\right)_{\mid \mathscr{F}(\mathscr{L}(G))}$ will be denoted also by $\eta_{G}$.

Proof. For a Lie group $H$ its Lie algebra $\mathscr{L}(H)$ consisting of all left invariant vector fields on $H$ we identify in the standard way with the tangent space $T_{1} H$.

Let $E_{1}, \ldots, E_{N}$ be a basis of $\mathscr{L}(G)$ and $a_{1}, \ldots, a_{K}$ be a basis of $A=\mathscr{F}(\mathbf{R})$. By Proposition $1.6(3) \mathscr{F}\left(E_{1}\right), \ldots, \mathscr{F}\left(E_{N}\right)$ is a basis of $\mathscr{F}(\mathscr{L}(G))$ over $A$. On the other hand by Proposition $2.6(3) E_{j}^{\left(a_{\nu}\right)}$ belong to $\mathscr{L}(\mathscr{F}(G))$ for $j=1, \ldots, N$ and $\nu=1$, $\ldots, K$. Of course, $\eta_{G}\left(a_{\nu} \mathscr{F}\left(E_{j}\right)\right)=E_{j}^{\left(a_{\nu}\right)}$. By Proposition 1.6

$$
\left[a_{\nu} \mathscr{F}\left(E_{j}\right), a_{\mu} \mathscr{F}\left(E_{i}\right)\right]=a_{\nu} a_{\mu}\left[\mathscr{F}\left(E_{j}\right), \mathscr{F}\left(E_{i}\right)\right]=a_{\nu} a_{\mu} \mathscr{F}\left(\left[E_{j}, E_{i}\right]\right)
$$

Thus

$$
\begin{aligned}
\eta_{G}\left(\left[a_{\nu} \mathscr{F}\left(E_{j}\right), a_{\mu} \mathscr{F}\left(E_{i}\right)\right]\right) & =\eta_{G}\left(a_{\nu} a_{\mu} \mathscr{F}\left(\left[E_{j}, E_{i}\right]\right)\right) \\
& =\left[E_{j}, E_{t}\right]^{\left(a_{\nu} a_{\mu}\right)} \\
& =\left[E_{j}^{\left(a_{\nu}\right)}, E_{i}^{\left(a_{\mu}\right)}\right] \\
& =\left[\eta_{G}\left(a_{\nu} \mathscr{F}\left(E_{j}\right)\right), \eta_{G}\left(a_{\mu} \mathscr{F}\left(E_{i}\right)\right)\right] .
\end{aligned}
$$

Since $\eta_{G}$ is a linear isomorphism, thus the proof is finished.

\section{Lifts of tensor fields of type $(1, k)$}

Let $\mathscr{F}$ be a product preserving functor and let $A=\mathscr{F}(\mathbf{R})$ be its Weil algebra. We define lifts of tensor fields of type $(1, k)$ from a manifold $M$ to $\mathscr{F}(M)$.

We interpret a tensor field $S$ of type $(1, k)$ on a manifold $M$ as a $k$-linear mapping $S: T M \times_{M} \cdots \times_{M} T M \rightarrow T M$ of the bundle product over $M$ of $k$ copies of the tangent bundle into $T M$ covering the identity on $M$. We recall that in this case the $k$-linearity means that restrictions of $S$ to fibres $\left(T M \times_{M} \cdots \times_{M} T M\right)_{x}$ $=T_{x} M \times \cdots \times T_{x} M \rightarrow T_{x} M$ is $k$-linear for all $x \in M$. Using $\eta_{X}: \mathscr{F}(T M) \rightarrow$ 
$T(\mathscr{F} M)$ we define a mapping $S^{C}: T(\mathscr{F} M) \times_{\mathscr{F}(M)} \cdots \times_{\mathscr{F}(M)} T(\mathscr{F} M) \rightarrow T(\mathscr{F} M)$ by

$$
S^{C}=\eta_{M} \circ \mathscr{F}(S) \circ\left(\eta_{M}^{-1} \times \cdots \times \eta_{M}^{-1}\right)
$$

called complete lift of $S$ from $M$ to $\mathscr{F}(M)$. From Propositions 1.9 and 1.7 we deduce that $S^{C}$ is a tensor field of type $(1, k)$ on $\mathscr{F}(M)$.

Let $a \in A$. For a tensor field $S$ of type $(1, k)$ on $M$ we define

$$
S^{(a)}=a \cdot S^{C}=\tilde{\Psi}\left(a, S^{C}\right),
$$

where the product "." is defined by (2.7). From properties of the product "." (see (2.8)) we obtain immediately that $S^{(a)}$ is a tensor field of type $(1, k)$ on $\mathscr{F}(M)$; it is called $a$-lift of $S$ from $M$ to $\mathscr{F}(M)$.

Let $\mathscr{T}_{k}^{1}(M)$ be the set of tensor fields of type $(1, k)$ on $M$. Let $S \in \mathscr{T}_{k}^{1}(M)$, $S^{\prime} \in \mathscr{T}_{k}^{1}(N)$ and $\varphi: M \rightarrow N$ be a smooth mapping. Tensor fields $S, S^{\prime}$ are $\varphi$-related if diagram (0.2) commutes. We have

PROPOSition 3.1. Let $\mathscr{F}$ be a product preserving functor and $A=\mathscr{F}(\mathbf{R})$ be its Weil algebra.

For every $a \in A$ the family of mappings $\mathscr{T}_{k}^{1}(M) \in S \rightarrow S^{(a)} \in \mathscr{T}_{k}^{1}(\mathscr{F} M)$ is a lifting, i.e. if $S \in \mathscr{T}_{k}^{1}(M)$ and $S^{\prime} \in \mathscr{T}_{k}^{1}(N)$ are $\varphi$-related, where $\varphi: M \rightarrow N$ is a smooth mapping, then $S^{(a)} \in \mathscr{T}_{k}^{1}(\mathscr{F} M)$ and $S^{\prime(a)} \in \mathscr{T}_{k}^{1}(\mathscr{F} M)$ are $\mathscr{F}(\varphi)$-related.

If $S$ is a tensor field of type $(1, k)$ and $a \in A$, then for all vector fields $X_{1}, \ldots$, $X_{k}$ on $M$ and all elements $a_{1}, \ldots, a_{k} \in A$ we have

$$
S^{(a)}\left(X_{1}^{\left(a_{1}\right)}, \ldots, X_{k}^{\left(a_{k}\right)}\right)=\left(S\left(X_{1}, \ldots, X_{k}\right)\right)^{\left(a a_{1} \ldots a_{k}\right)} .
$$

Let us observe that according to Proposition 2.9 formula (3.3) determines uniquely $S^{(a)}$.

Proof. Applying $\mathscr{F}, \eta_{M}$ and $\eta_{N}$ to diagram (0.2) we deduce the first part of the proposition.

To show formula (3.3) we apply $\mathscr{F}$ to the equality

$$
S\left(t_{1} X_{1}(x), \ldots, t_{k} X_{k}(x)\right)=t_{1} \ldots t_{k} S\left(X_{1}(x), \ldots, X_{k}(x)\right),
$$

where the left and the right sides are considered as mappings defined on $\mathbf{R} \times \cdots$ $\times \mathbf{R} \times M$, and we compose the induced mapping with the natural isomorphisms $\eta_{M}$ and $\eta_{M}^{-1}$. In consequence, we obtain

$$
S^{C}\left(a_{1} \cdot X_{1}^{C}, \ldots, a_{k} \cdot X_{k}^{C}\right)=a_{1} \ldots a_{k} \cdot S^{C}\left(X_{1}^{C}, \ldots, X_{k}^{C}\right) .
$$


By (3.2) it implies (3.3).

We will study algebraic properties of $a$-lifts of tensor fields. At first, we define an operation on tensor fields.

For two tensor fields $S, S^{\prime}$ on $M$ of type $(1, k)$ and $\left(1, k^{\prime}\right)$ respectively and for $i=1, \ldots, k$ we define a new tensor field $\rho^{i}\left(S, S^{\prime}\right)$ of type $\left(1, k+k^{\prime}-1\right)$ on $M$ by

$$
\begin{aligned}
& \rho^{i}\left(S, S^{\prime}\right)\left(v_{1}, \ldots, v_{k+k^{\prime}-1}\right) \\
& \quad=S\left(v_{1}, \ldots, v_{i-1}, S^{\prime}\left(v_{i}, \ldots, v_{i+k^{\prime}}\right), v_{i+k^{\prime}+1}, \ldots, v_{k+k^{\prime}-1}\right)
\end{aligned}
$$

for $v_{1}, \ldots, v_{k+k^{\prime}-1} \in T_{x} M, x \in M .^{3)}$

We have the following properties of $a$-lifts of tensor fields to $\mathscr{F}$.

Proposition 3.2. Let $\mathscr{F}$ be a product preserving functor, $A=\mathscr{F}(\mathbf{R})$ be its Weil algebra and let $a, b \in A$.

(1) If $S$ is a symmetric (respectively skew-symmetric) tensor field of type $(1, k)$ on $M$, then $S^{(a)}$ is a symmetric (respectively skew-symmetric) tensor field of type $(1, k)$ on $\mathscr{F}(M)$.

(2) For tensor fields $S, S^{\prime}$ of type $(1, k)$ on $M$ and reals $\alpha, \beta$ we have

$$
\begin{aligned}
\left(\alpha S+\beta S^{\prime}\right)^{(a)} & =\alpha S^{(a)}+\beta S^{(a)}, \\
S^{(\alpha a+\beta b)} & =\alpha S^{(a)}+\beta S^{(b)},
\end{aligned}
$$

(3) For tensor fields $S, S^{\prime}$ of type $(1, k)$ and $\left(1, k^{\prime}\right)$ respectively on $M$, and $i=$ $1, \ldots, k$ we have

$$
\left(\rho^{i}\left(S, S^{\prime}\right)\right)^{(a b)}=\rho^{i}\left(S^{(a)}, S^{(b)}\right),
$$

where $\rho^{i}\left(S, S^{\prime}\right)$ is defined by (3.4). Particularly, for two tensor fields $S, S^{\prime}$ of type $(1,1)$ on $M$ we obtain

$$
S^{(a)} S^{(b)}=\left(S S^{\prime}\right)^{(a b)} .
$$

(4) If $S$ is a tensor field of type $(1, k)$ on $M$ and $X$ is a vector field on $M$, then

$$
L_{X^{(a)}} S^{(b)}=\left(L_{X} S\right)^{(a b)},
$$

where $L$ denotes the Lie derivation.

(5) If $\delta_{M}: T M \rightarrow T M$ is the identity tensor, then $\left(\delta_{M}\right)^{(a)}=a \cdot \delta_{\mathscr{F}(M)}$. In particular, $\left(\delta_{M}\right)^{C}=\delta_{\mathscr{F}(M)}$.

3) If $S, S^{\prime}$ are tensor fields of type $(1,1)$, then $\rho^{1}\left(S, S^{\prime}\right)$ is the composition of $S: T M \rightarrow$ $T M$ and $S^{\prime}: T M \rightarrow T M$. 
(6) If $W(t)$ is a polynomial with real coefficients and $S$ is a tensor field of type $(1,1)$ on $M$, then $W\left(S^{C}\right)=(W(S))^{C}$.

Proof. The verification of parts (1)-(5) is obtained by similar methods. Part (6) is an immediate consequence of parts (2), (5) and (3). In order to present these methods we show part (3).

Let $X_{1}, \ldots, X_{k+k^{\prime}-1} \in \mathscr{K}(M)$ and $a_{1}, \ldots, a_{k+k^{\prime}-1} \in A=\mathscr{F}(\mathbf{R})$. Using (3.3) we have

$$
\begin{aligned}
\left(\rho^{i}(\right. & \left.\left.S^{(a)}, S^{\prime(b)}\right)\right)\left(X_{1}^{\left(a_{1}\right)}, \ldots, X_{k+k^{\prime}-1}^{\left(a_{\left.k+k^{\prime}-1\right)}\right)}\right. \\
& =S^{(a)}\left(\ldots \ldots, X_{i-1}^{\left(a_{i-1}\right)}, S^{(b)}\left(X_{i}^{\left(a_{i}\right)}, \ldots, X_{i+k^{\prime}}^{\left(a_{i+k^{\prime}}\right)}\right), X_{i+k^{\prime}+1}^{\left(a_{i+k^{\prime}+1}\right)}, \ldots \ldots\right) \\
& =S^{(a)}\left(\ldots \ldots, X_{i-1}^{\left(a_{i-1}\right)},\left(S^{\prime}\left(X_{i}, \ldots, X_{i+k^{\prime}}\right)\right)^{\left(b a_{i} \cdots a_{\left.i+k^{\prime}\right)}\right.}, X_{i+k^{\prime}+1}^{\left(a_{i+k^{\prime}+1}\right)}, \ldots \ldots\right) \\
& =\left(S\left(\ldots \ldots, X_{i-1}, S^{\prime}\left(X_{i}, \ldots, X_{i+k^{\prime}}\right), X_{i+k^{\prime}+1}, \ldots \ldots\right)\right)^{\left(a b a_{1} \cdots a_{k+k^{\prime}-1}\right)} \\
& =\left(\rho^{i}\left(S, S^{\prime}\right)\left(\ldots \ldots, X_{i-1}, X_{i}, \ldots, X_{i+k^{\prime}}, X_{i+k^{\prime}+1}, \ldots \ldots\right)\right)^{\left(a b a_{1} \cdots a_{k+k^{\prime}-1}\right)} \\
& =\left(\rho^{i}\left(S, S^{\prime}\right)\right)^{(a b)}\left(X_{1}^{\left(a_{1}\right)}, \ldots, X_{k+k^{\prime}-1}^{\left(a_{k+k^{\prime}-1}\right)}\right)
\end{aligned}
$$

Now by Proposition 2.9 we obtain part (3).

In the proof of (4) we use additionally the formula

$$
L_{X} S\left(X_{1}, \ldots, X_{k}\right)=\left[X, S\left(X_{1}, \ldots, X_{k}\right)\right]-\sum_{i=1}^{k} S\left(X_{1}, \ldots,\left[X, X_{i}\right], \ldots, X_{k}\right)
$$

and Proposition 2.6.

From the above proposition we obtain

COROLlary 3.3. Let $\mathscr{F}$ be a product preserving functor. If $t$ is an almost complex structure (respectively an almost tangent structure, an $f$-structure) on $M$, then $t^{C}$ is an almost complex structure (respectively an almost tangent structure, an $f_{\text {-structure) on }}$ $\mathscr{F}(M)$.

If $X$ is an infinitesimal transformation of $t$, then $X^{(a)}$ is an infinitesimal transformation of $t^{C}$ for every $a \in A=\mathscr{F}(\mathbf{R})$.

Proof. The first part follows from Proposition 3.2 (6) and the second one-from Proposition 3.2 (4).

The analogous properties were proved in the case of tangent bundle, $r$-order tangent bundle, $p^{r}$-velocities bundle by Yano, Ishihara and Morimoto (see [30], [18], [20], [22]). 
Next we propose to formulate the following important property.

Proposition 3.4. Let $\mathscr{F}$ be a product preserving functor and $A=\mathscr{F}(\mathbf{R})$ be the corresponding Weil algebra. If $t$ is a tensor field of type $(1,1)$ on $M$ and $a \in A$, then

$$
N_{t}(a)=\left(N_{t}\right)^{\left(a^{2}\right)} \text {, }
$$

where $N$ denotes the Nijenhuis tensor. In particular, we have

$$
N_{t^{c}}=\left(N_{t}\right)^{\mathrm{c}} \text {. }
$$

Proof. To prove this proposition we apply the similar methods as in Proposition 3.2 to the Nijenhuis tensor

$$
N_{t}(X, Y)=t^{2}[X, Y]-t[t X, Y]-t[X, t Y]+[t X, t Y] .
$$

Now we can prove

THEOREM 3.5. Let $\mathscr{F}$ be a product preserving functor. Let $t$ be an almost complex structure (respectively an almost tangent structure, an f-structure) on $M$. Then $t^{C}$ is a complex structure (respectively a tangent structure, an integrable f-structure) on $\mathscr{F}(M)$ if and only if $t$ is a complex structure (respectively a tangent structure, an integrable $f$-structure) on $M$.

Proof. For considered structures the integrability is equivalent to vanishing of the Nijenhuis tensor. Our theorem is a simple consequence of Proposition 3.4.

Let us observe that using Proposition 3.4 we can prove the analogous theorem for all geometric structures (defined by tensor fields $t$ of type $(1,1)$ ) for which the integrability is equivalent to vanishing of the Nijenhuis tensor.

\section{Lifts of tensor fields of type $(0, k)$ Lifts of $k$-forms}

In this paragraph we can define lifts of tensor fields of type $(0, k)$.

We interpret a tensor field $G$ of type $(0, k)$ on a manifold $M$ as a $k$-linear mapping $G: T M \times_{M} \cdots \times_{M} T M \rightarrow \mathbf{R}$ of the bundle product over $M$ of $k$ copies of the tangent bundle into $\mathbf{R}$. We recall that $k$-linearity means that its restrictions to fibres $\left(T M \times_{M} \cdots \times_{M} T M\right)_{x}=T_{x} M \times \cdots \times T_{x} M \rightarrow \mathbf{R}$ are $k$-linear for all $x$ $\in M$. Using the natural isomorphism $\eta_{M}: \mathscr{F}(T M) \rightarrow T(\mathscr{F} M)$ we define $\tilde{G}$ : $T(\mathscr{F} M) \times_{\mathscr{F}(M)} \cdots \times_{\mathscr{F}(M)} T(\mathscr{F} M) \rightarrow A$ by 


$$
\tilde{G}=\mathscr{F}(G) \circ\left(\eta_{M}^{-1} \times \cdots \times \eta_{M}^{-1}\right)
$$

$\tilde{G}$ is $k$-linear because $\mathscr{F}(G)$ is $k$-linear (see Proposition $1.7(3)$ ) and $\eta_{M}$ is linear (see Proposition 1.9).

Let $\lambda: A \rightarrow \mathbf{R}$ be a linear function. We define

$$
G^{(\lambda)}=\lambda \cdot \tilde{G} \text {. }
$$

It is a tensor field of type $(0, k)$ on $\mathscr{F}(M)$ called $\lambda$-lift of $G$ from $M$ to $\mathscr{F}(M)$.

Let $\mathscr{T}_{k}^{0}(M)$ be the space of tensor fields of type $(0, k)$ on $M$. We verify

Proposition 4.1. Let $\mathscr{F}$ be a product preserving functor, $A=\mathscr{F}(\mathbf{R})$ be its Weil algebra and let $\lambda: A \rightarrow \mathbf{R}$ be a linear function.

The family of mappings $\mathscr{T}_{k}^{0}(M) \ni G \rightarrow G^{(\lambda)} \in \mathscr{T}_{k}^{0}(\mathscr{F} M)$ is a lifting, i.e. if tensor fields $G \in \mathscr{T}^{0}(M)$ and $G^{\prime} \in \mathscr{T}_{k}^{0}(N)$ are $\varphi$-related, where $\varphi: M \rightarrow N$ is a smooth mapping, then $G^{(\lambda)}$ and $G^{\prime(\lambda)}$ are $\mathscr{F}(\varphi)$-related.

If $G$ is a tensor field of type $(0, k)$, then for all vector fields $X_{1}, \ldots, X_{k}$ on $M$ and all elements $a_{1}, \ldots, a_{k} \in A$ we have

$$
G^{(\lambda)}\left(X_{1}^{\left(a_{1}\right)}, \ldots, X_{k}^{\left(a_{k}\right)}\right)=\left(G\left(X_{1}, \ldots, X_{k}\right)\right)^{\left(\lambda \circ a_{a_{1} \ldots a_{k}}\right)},
$$

where $l_{a}: A \rightarrow A$ is given by $l_{a}(b)=a b$.

Proof. The first part of the proposition is clear. We verify (4.3) in a similar way as (3.3). From the equality $G\left(t_{1} X_{1}, \ldots, t_{k} X_{k}\right)=t_{1} \ldots t_{k} G\left(X_{1}, \ldots, X_{k}\right)$ we conclude that

$$
\tilde{G}\left(a_{1} \cdot X_{1}^{C}, \ldots, a_{k} \cdot X_{k}^{C}\right)=l_{a_{1} \ldots a_{k}} \circ \mathscr{F}\left(G\left(X_{1}^{C}, \ldots, X_{k}^{C}\right)\right) .
$$

Now, from (4.2) we obtain (4.3).

We have the following properties of $\lambda$-lifts.

Proposition 4.2. Let $\mathscr{F}$ be a product preserving functor, $A=\mathscr{F}(\mathbf{R})$ be its Weil algebra, $\lambda, \lambda^{\prime}: A \rightarrow \mathbf{R}$ be linear functions and let $a \in A$.

(1) If $G, G^{\prime}$ are tensor fields of type $(0, k)$ on $M$ and $\alpha, \beta$ are reals, then

$$
\begin{aligned}
\left(\alpha G+\beta G^{\prime}\right)^{(\lambda)} & =\alpha G^{(\lambda)}+\beta G^{\prime(\lambda)}, \\
G^{\left(\alpha \lambda+\beta \lambda^{\prime}\right)} & =\alpha G^{(\lambda)}+\beta G^{\left(\lambda^{\prime}\right)} .
\end{aligned}
$$

(2) Let $G$ be a tensor field of type $(0, k)$ on $M$. If $G$ is symmetric (respectively skew-symmetric) then $G^{(\lambda)}$ is also symmetric (respectively skew-symmetric). 
(3) If $G$ is a tensor field of type $(0, k)$ on $M$ and $S$ is a tensor field of type $(1, s)$ on $M$, then for $i=1, \ldots, k$ we have

$$
\rho^{i}\left(G^{(\lambda)}, S^{(a)}\right)=\left(\rho^{i}(G, S)\right)^{\left(\lambda \circ l_{a}\right)},
$$

where $\rho^{i}(G, S)$ is a tensor field of type $(0, k+s-1)$ given by ${ }^{4)}$

$$
\begin{aligned}
& \rho^{i}(G, S)\left(X_{1}, \ldots, X_{k+s-1}\right) \\
& \quad=G\left(X_{1}, \ldots, X_{i-1}, S\left(X_{i}, \ldots, X_{i+s}\right), X_{i+s+1}, \ldots, X_{s+k-1}\right)
\end{aligned}
$$

for vector fields $X_{1}, \ldots, X_{k+s-1}$ on $M$.

(4) If $G$ is a tensor field of type $(0, k)$ on $M$ and $X$ is a vector field on $M$, then

$$
L_{X^{(a)}} G^{(\lambda)}=\left(L_{X} G\right)^{\left(\lambda \circ l_{a}\right)} .
$$

Proof. The verification is similar to the proof of Proposition 3.2. We use (4.3) instead of (3.3) and (2.3) instead of (2.13).

From Proposition 4.2 (2) we obtain that if $\omega$ is a $k$-form on $M$, then its $\lambda$-lift $\omega(\lambda)$ is a $k$-form on $\mathscr{F}(M)$. We prove also:

Proposition 4.3. Let $\mathscr{F}$ be a product preserving functor and $A=\mathscr{F}(\mathbf{R})$ be its Weil algebra. If $\omega$ is a $k$-form on $M$ and $\lambda: A \rightarrow \mathbf{R}$ is a linear function, then

$$
d \omega^{(\lambda)}=(d \omega)^{(\lambda)} \text {. }
$$

Proof. To show this proposition we apply the similar methods as in Proposition 3.2. We use the formula

$$
\begin{aligned}
d \omega\left(X_{0}, \ldots, X_{k}\right) & =\frac{1}{k+1}\left\{\sum_{i=0}^{k}(-1)^{i} X_{i}\left(\omega\left(X_{0}, \ldots, \hat{X}_{i}, \ldots, X_{k}\right)\right)\right. \\
& \left.+\sum_{0 \leq i<j \leq k}(-1)^{i+j} \omega\left(\left[X_{i}, X_{j}\right], X_{0}, \ldots, \hat{X}_{i}, \ldots, \hat{X}_{j}, \ldots, X_{k}\right)\right\} .
\end{aligned}
$$

As an immediate consequence of Propositions 4.3 and 4.2 (4) we obtain

Corollary 4.4. Let $\omega$ be a $k$-form and $\lambda: A=\mathscr{F}(\mathbf{R}) \rightarrow \mathbf{R}$ be a linear function.

If $\omega$ is closed, then its $\lambda$-lift $\omega^{(\lambda)}$ is also closed.

If $X$ is an infinitesimal transformation of $\omega$ and $a \in A$, then $X^{(a)}$ is an infinitesimal transformation of $\omega^{(\lambda)}$.

4) $\rho^{2}(G, S)$ is a contraction of $G \otimes S$. 
If $g$ is a pseudo-Riemannian tensor on $M$, then for each linear function $\lambda$ : $A \rightarrow \mathbf{R}$ its $\lambda$-lift $g^{(\lambda)}$ is a quadratic form on $\mathscr{F}(M)$ but, in general, $g^{(\lambda)}$ is not non-singular. We prove the following proposition about the signature of $g^{(\lambda)}$. Namely we have

Proposition 4.5. Let $\lambda: A \rightarrow \mathbf{R}$ be a linear function on the Weil algebra $A=$ $\mathscr{F}(\mathbf{R})$ of a product preserving functor $\mathscr{F}$ and let $\left(p_{\lambda}^{+}, p_{\lambda}^{-}\right)$be the signature ${ }^{5)}$ of the symmetric 2-form $A \times A \ni(a, b) \rightarrow \lambda(a b) \in \mathbf{R}$. If $g$ is a symmetric tensor field of type $(0,2)$ on $M$ with a constant signature $\left(p_{g}^{+}, p_{g}^{-}\right)$, then $g^{(\lambda)}$ is a symmetric tensor field of type $(0,2)$ on $\mathscr{F}(M)$ with a constant signature $\left(p_{g}^{+} p_{\lambda}^{+}+p_{g}^{-} p_{\lambda}^{-}, p_{g}^{-} p_{\lambda}^{+}+p_{g}^{+} p_{\lambda}^{-}\right)$.

Proof. We fix a point of $M$. There is a basis $X_{1}, \ldots, X_{n}$ of vector fields on some neighborhood $U$ of the fixed point such that

$$
g\left(X_{i}, X_{j}\right)= \begin{cases}1, & \text { for } i=j=1, \ldots, p_{g}^{+} \\ -1, & \text { for } i=j=p_{g}^{+}+1, \ldots, p_{g}^{+}+p_{g}^{-} \\ 0, & \text { in the other cases. }\end{cases}
$$

There is a basis $a_{1}, \ldots, a_{k}$ of $A$ such that

$$
\lambda\left(a_{\nu} a_{\mu}\right)= \begin{cases}1, & \text { for } \nu=\mu=1, \ldots, p_{\lambda}^{+} \\ -1, & \text { for } \nu=\mu=p_{\lambda}^{+}+1, \ldots, p_{\lambda}^{+}+p_{\lambda}^{-} \\ 0, & \text { in the other cases. }\end{cases}
$$

Now, by (4.3) we obtain

$$
\begin{aligned}
g^{(\lambda)}\left(X_{i}^{\left(a_{\nu}\right)}, X_{j}^{\left(a_{\mu}\right)}\right) & =\left(g\left(X_{i}, X_{j}\right)\right)^{\left(\lambda \circ l_{\left.a_{\nu} a_{\mu}\right)}\right.} \\
& =\lambda\left(a_{\nu} a_{\mu} \mathscr{F}\left(g\left(X_{i}, X_{j}\right)\right)\right) .
\end{aligned}
$$

Since $g\left(X_{i}, X_{j}\right)$ is a constant function, thus $\mathscr{F}\left(g\left(X_{i}, X_{j}\right)\right)$ is also a constant function and $\mathscr{F}\left(g\left(X_{i}, X_{j}\right)\right)=g\left(X_{i}, X_{j}\right)$. Now, by the linearity of $\lambda$ we obtain

$$
\begin{aligned}
g^{(\lambda)}\left(X_{i}^{\left(a_{\nu}\right)}, X_{j}^{\left(a_{\mu}\right)}\right)=g\left(X_{i}, X_{j}\right) \lambda\left(a_{\nu} a_{\mu}\right) \\
\quad= \begin{cases}1, & \text { for } i=j=1, \ldots, p_{g}^{+}, \nu=\mu=1, \ldots, p_{\lambda}^{+} \\
-1, & \text { for } i=j=1, \ldots, p_{g}^{+}, \nu=\mu=p_{\lambda}^{+}+1, \ldots, p_{\lambda}^{+}+p_{\lambda}^{-} \\
-1, & \text { for } i=j=p_{g}^{+}+1, \ldots, p_{g}^{+}+p_{g}^{-}, \nu=\mu=1, \ldots, p_{\lambda}^{+} \\
1, & \text { for } i=j=p_{g}^{+}+1, \ldots, p_{g}^{+}+p_{g}^{-}, \nu=\mu=p_{\lambda}^{+}+1, \ldots, p_{\lambda}^{+}+p_{\lambda}^{-} \\
0, & \text { in the other cases. }\end{cases}
\end{aligned}
$$

5) The signature of a symmetric form is a pair $(p, q)$, where $p$ is the number of positive elements and $q$ is the number of negative numbers in a diagonal matrix of the form. 
It means that $g^{(\lambda)}$ is of constant signature $\left(p_{g}^{+} p_{\lambda}^{+}+p_{g}^{-} p_{\lambda}^{-}, p_{g}^{-} p_{\lambda}^{+}+p_{g}^{+} p_{\lambda}^{-}\right)$because the family $X_{i}^{\left(a_{\nu}\right)}, i=1, \ldots, n, \nu=1, \ldots, K$ is a basis of vector fields on $\mathscr{F}(U)$.

We can apply the above proposition to lift a (pseudo-)Riemannian metric.

Let us observe that for a Riemannian metric $g$ on $M$ its lift $g^{(\lambda)}$ is never a Riemannian structure on $\mathscr{F}(M)$. It is an immediate consequence of the following lemma

LEMma 4.6. If $\mathscr{F}$ is a product preserving functor such that $\operatorname{dim} A>1^{6)}$, then there is no linear function $\lambda: A \rightarrow \mathbf{R}$ such that the symmetric form $A \times A \ni$ $(a, b) \rightarrow \lambda(a b) \in \mathbf{R}$ is positive definite.

Proof. The assumption $\operatorname{dim} A>1$ implies that there is $a \in A$ such that $a \neq 0$ and $a^{2}=0$. Now, by the linearity, we have $\lambda\left(a^{2}\right)=0$, i.e. the considered symmetric form is not positive definite.

Let $g$ be a pseudo-Riemannian metric on $M$. Its $\lambda$-lift $g^{(\lambda)}$ is a pseudoRiemannian metric on $\mathscr{F}(M)$ if and only if $\lambda: A \rightarrow \mathbf{R}$ is a linear function such that the symmetric form $A \times A \ni(a, b) \rightarrow \lambda(a b) \in \mathbf{R}$ is non-singular. Thus, to lift pseudo-Riemannian metrics we need to find a linear function $\lambda$ with this property. In general, there are no such linear functions $\lambda$ because we have.

Proposition 4.7. Let $\mathscr{F}$ be a product preserving functor, $A$ be its Weil algebra and $\operatorname{dim} A>1$. Let $E$ be a vector subspace of all elements $a \in A$ such that for each nilpotent element $u \in A$ we have au $=0^{7)}$. If there exists a linear function $\lambda: A \rightarrow \mathbf{R}$ such that the symmetric form $(a, b) \rightarrow \lambda(a b)$ is non-singular, then $\operatorname{dim} E=1$.

Proof. Let $a \in E$ and $a \neq 0$. Since the symmetric form $(a, b) \rightarrow \lambda(a b)$ is non-singular thus there exists $b \in A$ such that $\lambda(a b) \neq 0 . b$ can be written in the form $b=\alpha 1+u$, where $\alpha \in \mathbf{R}$ and $u$ is a nilpotent element because $A=\mathbf{R} \cdot 1+$ $N$, where $N$ is the subalgebra of nilpotent elements. Now from $0 \neq \lambda(a b)=$ $\alpha \lambda(a)$ we obtain $\lambda(a) \neq 0$. It implies that $\lambda \mid E: E \rightarrow \mathbf{R}$ is a monomorphism. Hence $\operatorname{dim} E \leq 1$. This finishes the proof.

6) $\operatorname{dim} A=1$ if and only if $\mathscr{F}$ is the identity functor, i.e. $\mathscr{F}(M)=M$ and $\mathscr{F}(\varphi)=\varphi$.

7) From the characterization of Weil algebras it follows that $\operatorname{dim} E \geq 1$. 


\section{Complete lift of linear connections}

In this section we will study lifts of linear connections from a manifold $M$ to linear connections on $\mathscr{F}(M)$. We start from very general situation of connections in principal fibre bundles.

We recall that for a Lie group $G$ there is a natural isomorphism $\eta_{G}$ : $\mathscr{F}(\mathscr{L} G) \rightarrow \mathscr{L}(\mathscr{F} G)$ of Lie algebra (see Proposition 1.10$)$. We prove the following proposition about fundamental vector fields on a manifold associated with an action of a Lie group. Namely we have

Proposition 5.1. Let $\mathscr{F}$ be product preserving functor. Let $P$ be a $G$-space and for an element $U \in \mathscr{L}(G)$ let $U^{*}$ be the fundamental vector field on $M$ defined by $U$. If $L: P \times \mathscr{L}(G) \rightarrow T P$ is given by $L(p, U)=U_{p}^{*}$, then for an element $\bar{U}$ of $\mathscr{L}(\mathscr{F} G)$ the corresponding fundamental vector field $\bar{U}^{*}$ on $\mathscr{F}(P)$ is given by $\bar{U}_{y}^{*}=$ $\left(\eta_{P} \circ \mathscr{F}(L)\right)\left(y, \eta_{G}^{-1}(\bar{U})\right)$.

Proof. If $\Lambda: P \times G \rightarrow P$ is the action of $G$ on $P$, then, by Proposition 1.8 (2), $\mathscr{F}(\Lambda)$ defines the action of $\mathscr{F}(G)$ on $\mathscr{F}(P)$. Let us observe that $U_{p}^{*}=d \Lambda\left(0_{p}, U\right)$, where $0_{p}$ is zero in $T_{p} P$. Thus we have $L=d \Lambda \circ\left(0_{P} \times \operatorname{id}_{\mathscr{L}(G)}\right)$, where $0_{P}: P \rightarrow T P$ is the zero vector field.

Let $\bar{L}: \mathscr{F}(P) \times \mathscr{L}(\mathscr{F} G) \rightarrow T(\mathscr{F} P)$ be the corresponding mapping for $\mathscr{F}(P)$. Since $\bar{L}=d(\mathscr{F} \Lambda) \circ\left(0_{\mathscr{F}(P)} \times \mathrm{id}_{\mathscr{L}(\mathscr{F}(G))}\right)$, thus by Propositions 1.9 and 1.10 (3) we conclude

$$
\begin{aligned}
\bar{L} & =\eta_{P} \circ \mathscr{F}(d \Lambda) \circ\left(\eta_{P}^{-1} \times \eta_{G}^{-1}\right) \circ\left(0_{\mathscr{F}(P)} \times \operatorname{id}_{\mathscr{L}(\mathscr{F} G)}\right) \\
& =\eta_{P} \circ \mathscr{F}(d \Lambda) \circ\left(\eta_{P}^{-1} \circ 0_{\mathscr{F}(P)} \times \eta_{G}^{-1}\right) .
\end{aligned}
$$

The composition $\eta_{P}^{-1} \circ O_{\mathscr{F}(P)}$ is zero section of $\mathscr{F}(T P) \rightarrow \mathscr{F}(P)$, in consequence, $\eta_{P}^{-1} \circ O_{\mathscr{F}(P)}=\mathscr{F}\left(0_{P}\right)$. Now we obtain

$$
\begin{aligned}
\bar{L} & =\eta_{P} \circ \mathscr{F}(d \Lambda) \circ\left(\mathscr{F}\left(0_{P}\right) \times \eta_{G}^{-1}\right) \\
& =\eta_{P} \times \mathscr{F}\left(d \Lambda \circ\left(0_{P} \times \mathrm{id}_{\mathscr{L}(G)}\right)\right) \circ\left(\mathrm{id}_{\mathscr{F}(P)} \times \eta_{G}^{-1}\right) \\
& =\eta_{P} \times \mathscr{F}(L) \circ\left(\mathrm{id}_{\mathscr{F}(P)} \times \eta_{G}^{-1}\right) .
\end{aligned}
$$

It implies

$$
\bar{U}_{y}^{*}=\bar{L}(y, \bar{U})=\left(\eta_{P} \circ \mathscr{F}(L)\right)\left(y, \eta_{G}^{-1}(\bar{U})\right)
$$

Now we define a lift of connections in principal fibre bundles. Let $\omega: T P \rightarrow$ $\mathscr{L}(G)$ be a connection form on a principal fibre bundle $P(M, G)$. Using the 
induced mapping $\mathscr{F}(\omega): \mathscr{F}(T P) \rightarrow \mathscr{F}(\mathscr{L} G)$ and the canonical isomorphisms $\eta_{P}: \mathscr{F}(T P) \rightarrow T \mathscr{F}(P), \eta_{G}: \mathscr{F}(\mathscr{L} G) \rightarrow \mathscr{L}(\mathscr{F} G)$ we define $\omega^{c}: T \mathscr{F}(P) \rightarrow \mathscr{L}(\mathscr{F} G)$ by

$$
\omega^{c}=\eta_{G} \circ \mathscr{F}(\omega) \circ \eta_{P}^{-1} .
$$

$\omega^{c}$ is called complete lift of $\omega$ from $P$ to $\mathscr{F}(P)$.

We have (see [26]).

Proposition 5.2. Let $\mathscr{F}$ be a product preserving functor. If $\omega: T P \rightarrow \mathscr{L}(G)$ is a connection in a principal fibre bundle $P(M, G)$, then the complete lift $\omega^{c}: T \mathscr{F}(P) \rightarrow$ $\mathscr{L}(\mathscr{F} G)$ is a connection in the principal fibre bundle $\mathscr{F}(P)(\mathscr{F}(M), \mathscr{F}(G))$.

Proof. We define $r: G \times T P \rightarrow T P$ by $r(\xi, v)=d R_{\xi}(v)=\left(d R \circ\left(0_{G} \times\right.\right.$ $\left.\left.\operatorname{id}_{T P}\right)\right)(\xi, v)$, where $R(p, \xi)=R_{\xi}(p)$ is the right translation on $P$, and $\operatorname{Ad}: G \times$ $\mathscr{L}(G) \rightarrow \mathscr{L}(G)$ by $\operatorname{Ad}(\xi, y)=\mathscr{L}\left(\operatorname{ad}_{\xi}\right)(y)=\left(d \mathrm{ad} \circ\left(0_{G} \times \mathrm{id}_{T_{1} G}\right)\right)(\xi, y)$, where $\operatorname{ad}(\xi, \zeta)=\operatorname{ad}_{\xi}(\zeta)$ is the adjoint action of $G$ on $G$. Let $\bar{r}: \mathscr{F}(G) \times T(\mathscr{F} P) \rightarrow$ $T(\mathscr{F} P)$ and $\overline{\mathrm{Ad}}: \mathscr{F}(G) \times \mathscr{L}(\mathscr{F} G) \rightarrow \mathscr{L}(\mathscr{F} G)$ be the corresponding mappings for the principal fibre bundle $\mathscr{F}(P)$ and the Lie group $\mathscr{F}(G)$. Similarly to (5.1) we verify

$$
\begin{aligned}
\overline{\mathrm{Ad}} & =\eta_{G} \circ \mathscr{F}(\mathrm{Ad}) \circ\left(\mathrm{id}_{\mathscr{F}(G)} \times \eta_{G}^{-1}\right) \\
\bar{r} & =\eta_{P} \circ \mathscr{F}(r) \circ\left(\mathrm{id}_{\mathscr{F}(G)} \times \eta_{G}^{-1}\right) .
\end{aligned}
$$

Since $\omega$ is a connection, thus we have $\omega^{\circ} d R_{\xi}=\mathscr{L}\left(\operatorname{ad}_{\xi-1}\right) \circ \omega$ and $\omega\left(U^{*}\right)=U$ for all $U \in \mathscr{L}(G)$. Using our notations we can write equivalently these formulas in the form

$$
\begin{aligned}
& \omega \circ r=\operatorname{Ad} \bullet(k \times \omega) \\
& \omega \circ L=p_{2}
\end{aligned}
$$

where $k: G \rightarrow G$ is defined by $k(\xi)=\xi^{-1}$ and $p_{2}: P \times \mathscr{L}(G) \rightarrow \mathscr{L}(G)$ is the standard projection.

To show that $\omega^{c}$ is a connection in $\mathscr{F}(P)$ we need to verify

$$
\begin{aligned}
\omega^{c} \circ \bar{r} & =\overline{\mathrm{Ad}} \circ\left(\bar{k} \times \omega^{c}\right) \\
\omega^{c} \circ \bar{L} & =\overline{p_{2}}
\end{aligned}
$$

where $\bar{k}=\mathscr{F}(k): \mathscr{F}(G) \rightarrow \mathscr{F}(G)$ and $\bar{p}_{2}: \mathscr{F}(P) \times \mathscr{L}(\mathscr{F}(G)) \rightarrow \mathscr{L}(\mathscr{F}(G))$ is the standard projection. $\overline{p_{2}}$ is given by

$$
\overline{p_{2}}=\eta_{G} \circ \mathscr{F}\left(p_{2}\right) \circ\left(\mathrm{id}_{\mathscr{F}(P)} \times \eta_{G}^{-1}\right) .
$$


We will verify (5.7). From (5.5), by (5.3) and (5.4) we obtain

$$
\begin{aligned}
\omega^{c} \circ \bar{r} & =\left(\eta_{G} \circ \mathscr{F}(\omega) \circ \eta_{P}^{-1}\right) \circ\left(\eta_{P} \circ \mathscr{F}(r) \circ\left(\mathrm{id}_{\mathscr{F}(G)} \times \eta_{P}^{-1}\right)\right) \\
& =\eta_{G} \circ \mathscr{F}(\omega \circ r) \circ\left(\mathrm{id}_{\mathscr{F}(G)} \times \eta_{P}^{-1}\right) \\
& =\eta_{G} \circ \mathscr{F}(\mathrm{Ad}) \circ(\mathscr{F}(k) \times \mathscr{F}(\omega)) \circ\left(\mathrm{id}_{\mathscr{F}(G)} \times \eta_{P}^{-1}\right) \\
& =\overline{\operatorname{Ad}} \circ\left(\mathrm{id}_{\mathscr{F}(G)} \times \eta_{G}\right) \circ(\bar{k} \times \mathscr{F}(\omega)) \circ\left(\mathrm{id}_{\mathscr{F}(G)} \times \eta_{P}^{-1}\right) \\
& =\overline{\operatorname{Ad}} \circ\left(\bar{k} \times \omega^{c}\right) .
\end{aligned}
$$

The formula (5.7) is proved. Analogously by (5.1), (5.6) and (5.9) we obtain (5.8).

From definition of the complete lift of connections we can deduce

PROPOSITION 5.3. Let $\mathscr{F}$ be a product preserving functor and $\omega: T P \rightarrow \mathscr{L}(G)$ be a connection in a principal fibre bundle $P(M, G)$.

(1) If $\Gamma$ and $\bar{\Gamma}$ are the horizontal distributions for $\omega$ and $\omega^{c}$ respectively ${ }^{8)}$, then $\bar{\Gamma}$ $=\eta_{P}(\mathscr{F}(\Gamma))$.

(2) If

$$
\begin{aligned}
& h^{\omega}: T P \rightarrow T P, \quad h^{\omega^{c}}: T \mathscr{F}(P) \rightarrow T \mathscr{F}(P) \\
& v^{\omega}: T P \rightarrow T P, \quad v^{\omega^{c}}: T \mathscr{F}(P) \rightarrow T \mathscr{F}(P)
\end{aligned}
$$

are the horizontal and vertical projections for $\omega$ and $\omega^{c}$ respectively, then the diagrams
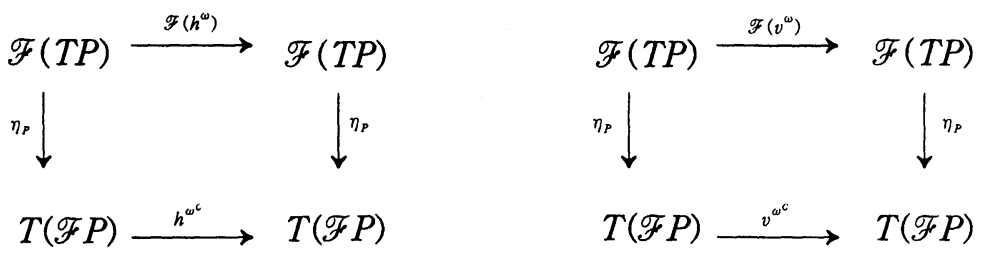

commute.

(3) For a vector field $X$ let $X^{\omega}$ denote the horizontal lift of $X$ to $P$ with respect to $\omega^{9)}$. If $a \in A$, where $A=\mathscr{F}(\mathbf{R})$ is the Weil algebra of $\mathscr{F}$, then we have

$$
\left(X^{(a)}\right)^{\omega^{c}}=\left(X^{\omega}\right)^{(a)} \text {. }
$$

8) They are considered as submanifolds of $T P$ and $T \mathscr{F}(P)$ respectively.

9) $X^{\omega}$ is the unique vector fields on $P$ such that $\omega^{\circ} X^{\omega}=0, d \pi^{\circ} X^{\omega}=X^{\circ} \pi$, where $\pi: P$ $\rightarrow M$ is the bundle projection. 
Proof. Part (1) is an immediate consequence of the definition of $\omega^{c}$ and of formula (1.4). Part (2) follows from (1) and the following equality for the vertical distributions $V(\mathscr{F} P)=\eta_{P}(\mathscr{F}(V P))$ which is a consequence of (1.4).

Part (3) is a consequence of the definitions of $\omega^{c}, X^{(a)}$, of the fact that $\mathscr{F}(\pi): \mathscr{F}(P) \rightarrow \mathscr{F}(M)$ is the bundle projection and of the last formula from (2.8) for $\varphi=\pi$.

The constructed complete lift of connection satisfies the naturality condition. In order to formulate this property we introduce a definition of $f$-related connections.

Let $f: P \rightarrow P^{\prime}$ be a homomorphism of principal fibre bundles and let $\rho_{f}: G$ $\rightarrow G^{\prime}$ be the corresponding Lie group homomorphism. Connections $\omega: T P \rightarrow$ $\mathscr{L}(G)$ and $\omega^{\prime}: T P^{\prime} \rightarrow \mathscr{L}\left(G^{\prime}\right)$ are called $f$-related if the following diagram

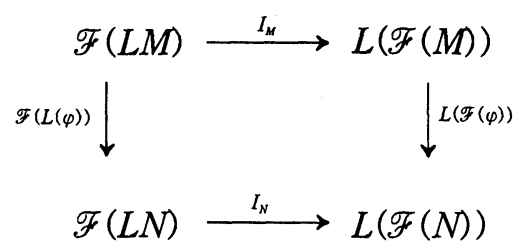

commutes.

Now we have (cf. Pogoda [26]).

Proposition 5.4. Let $\mathscr{F}$ be a product preserving functor. Let $f: P(M, G) \rightarrow$ $P^{\prime}\left(M^{\prime}, G^{\prime}\right)$ be a homomorphism of principal fibre bundles and let $\omega, \omega^{\prime}$ be connections in $P(M, G)$ and $P^{\prime}\left(M^{\prime}, G^{\prime}\right)$ respectively. If $\omega$ and $\omega^{\prime}$ are $f$-related, then $\omega^{c}$ and $\omega^{\prime c}$ are $\mathscr{F}(f)$-related.

Proof. Applying $\mathscr{F}$ to diagram (5.10) and using $\eta_{P}, \eta_{P^{\prime}}, \eta_{G}, \eta_{G^{\prime}}$ we complete the proof.

Proposition 5.4 means that the family of mappings $\omega \rightarrow \omega^{c}$ is "gauge-natural" transformation (see [3]).

To transform the obtained results for linear connections we will need some following properties of linear bundles.

Proposition 5.5. Let $\mathscr{F}$ be a product preserving functor and $A=\mathscr{F}(\mathbf{R})$ be its Weil algebra. 
For every manifold $M$ there exists one and only one monomorphism

$$
I_{M}: \mathscr{F}(L M) \rightarrow L(\mathscr{F}(M))
$$

of principal fibre bundles covering the identity on $\mathscr{F}(M)$ and with the inclusion $I: \mathscr{F}\left(G L\left(\mathbf{R}^{n}\right)\right) \rightarrow G L\left(\mathscr{F}\left(\mathbf{R}^{n}\right)\right)$ given in Proposition 1.8 (2) such that for each chart $(U, \varphi)$ on $M$ we have $I_{M} \circ \mathscr{F}\left(\sigma_{\varphi}\right)=\sigma_{\mathscr{F}(\varphi)}$, where $\sigma_{\varphi}: U \rightarrow L M$ and $\sigma_{\mathscr{F}(\varphi)}: \mathscr{F}(U) \rightarrow$ $L(\mathscr{F} M)$ are local sections associated with $\varphi$ and $\mathscr{F}(\varphi)$ respectively.

The family $\left\{I_{M}\right\}$ is natural, i.e. for every embedding $\varphi: M \rightarrow N$ of two $n$-dimensional manifolds $M, N$ the diagram

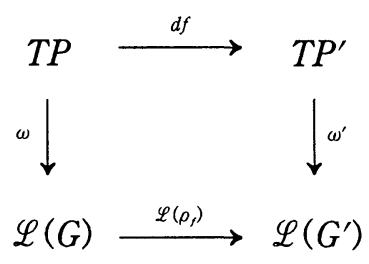

commutes, where $L(\varphi): L M \rightarrow L N$ is the induced mapping.

Proof. We choose the canonical mapping $K_{M}: L M \times \mathbf{R}^{n} \rightarrow T M, K_{M}(l, v)=$ $l(v)$. Let us define $I_{M}: \mathscr{F}(L M) \rightarrow L(\mathscr{F} M)$ by

$$
I_{M}(\bar{l})(\bar{v})=\left(\eta_{M} \circ \mathscr{F}\left(K_{M}\right)\right)(\bar{l}, \bar{v}),
$$

where $\bar{l} \in \mathscr{F}(L M), \bar{v} \in A^{n}$ and $\eta_{M}$ is defined in Proposition 1.9. Since $K_{M}(l X, v)=K_{M}(l, X v)$ we obtain $\mathscr{F}\left(K_{M}\right)(\bar{l} \bar{X}, \bar{v})=\mathscr{F}\left(K_{M}\right)(\bar{l}, \bar{X} \bar{v})$ for all $\bar{l} \in$ $\mathscr{F}(L M), \bar{X} \in \mathscr{F}\left(G L\left(\mathbf{R}^{n}\right)\right) \subset G L\left(\mathscr{F}\left(\mathbf{R}^{n}\right)\right)$ and $\bar{v} \in A^{n}$, where the inclusion $I$ is described in Proposition 1.8 (2). Therefore

$$
\left(I_{M}(\bar{l} \bar{X})\right)(\bar{v})=\left(I_{M}(\bar{l})\right)(\bar{X} \bar{v})=\left(I_{M}(\bar{l}) \bar{X}\right)(\bar{v}),
$$

i.e. $I_{M}$ is a principal fibre bundle homomorphism. Since the corresponding Lie group homomorphism is the inclusion $I, I_{M}$ is a pricipal fibre bundle monomorphism.

If $\varphi$ is a chart on $M$, then $K_{M}\left(\sigma_{\varphi}(x), v\right)=d \varphi^{-1}(\varphi(x), v)$ after the standard identification $T \mathbf{R}^{n}$ with $\mathbf{R}^{n} \times \mathbf{R}^{n}$. Using $\mathscr{F}$ and (5.12) we obtain

$$
\begin{aligned}
I_{M}\left(\mathscr{F}\left(\sigma_{\varphi}\right)(\bar{x})\right)(\bar{v}) & =\left(\eta_{M} \circ \mathscr{F}\left(K_{M}\right)\right)\left(\mathscr{F}\left(\sigma_{\varphi}\right)(\bar{x}), \bar{v}\right) \\
& =\left(\eta_{M} \circ \mathscr{F}\left(d \varphi^{-1}\right)\right)(\mathscr{F}(\varphi)(\bar{x}), \bar{v}) \\
& =d \mathscr{F}\left(\varphi^{-1}\right)(\mathscr{F}(\varphi)(\bar{x}), \bar{v}) \\
& =\sigma_{\mathscr{F}(\varphi)}(\bar{x})(\bar{v}) .
\end{aligned}
$$


Thus $I_{M} \circ \mathscr{F}\left(\sigma_{\varphi}\right)=\sigma_{\mathscr{F}(\varphi)}$.

We will study lifts of linear connections from a manifold $M$ to linear connections on $\mathscr{F}(M)$.

Let $\omega: T L M \rightarrow g l(n, \mathbf{R})$ be a connection form on the bundle of linear frames (a linear connection on $M$ ), where $g l(n, \mathbf{R})$ denotes the Lie algebra of the linear group and $n=\operatorname{dim} M$. If we apply our construction to a linear connection $\omega$, we obtain a connection $\omega^{C}: T \mathscr{F}(L M) \rightarrow \mathscr{L}\left(\mathscr{F} G L\left(\mathbf{R}^{n}\right)\right)$ in $\mathscr{F}(L M)$. Using the inclusion $I_{M}: \mathscr{F}(L M) \rightarrow L(\mathscr{F}(M))$ from Proposition 5.5 we prolong $\omega^{C}$ to one and only one linear connection $T L(\mathscr{F}(M)) \rightarrow G L\left(\mathscr{F}\left(\mathbf{R}^{n}\right)\right)$ denoted also by $\omega^{c}$ and called also the complete lift.

We prove the following property of $\omega^{c}$.

Proposition 5.6. Let $\mathscr{F}$ be a product preserving functor.

Let $\omega: T L M \rightarrow g l(n, \mathbf{R})$ be a linear connection on $M$. If $\nabla$ and $\nabla^{C}$ are the covariant derivations of $\omega$ and $\omega^{c}$ respectively, then for all vector fields $X, Y$ on $M$ and all $a, b \in A=\mathscr{F}(\mathbf{R})$ we have

$$
\nabla_{X^{(a)}}^{C} Y^{(b)}=\left(\nabla_{X} Y\right)^{(a b)} .
$$

$\nabla^{C}$ is the unique linear connection on $\mathscr{F}(M)$ satisfying equality (5.13) for all vector fields $X, Y$ on $M$ and all $a, b \in A$.

If $\nabla$ and $\nabla^{\prime}$ are two $\varphi$-related connections on $n$-dimensional manifolds $N$ and $M$ respectively, where $\varphi: M \rightarrow N$ is an embedding, then $\nabla^{C}$ and $\nabla^{\prime C}$ are $\mathscr{F}(\varphi)$-related.

Proof. For a vector field $X$ on $M$ let $X^{\sim}: L M \rightarrow \mathbf{R}^{n}$ be the corresponding mapping such that for $p \in L M$ the value $X^{\sim}(p)$ are coordinates of $X(\pi(p))$ in the basis $p$. Now we have (see [2] or [13])

$$
\left(\nabla_{X} Y\right)^{\sim}=X^{\omega}\left(Y^{\sim}\right) .
$$

Since $Y_{\pi(l)}=K_{M}\left(l, Y^{\sim}(l)\right)$, where $K_{M}: L M \times \mathbf{R}^{n} \rightarrow T M$ is the canonical mapping, then

$$
Y_{\mathscr{F}(\pi) \bar{l})}^{C}=\left(\eta_{M} \circ \mathscr{F}\left(K_{M}\right)\right)\left(\bar{l}, \mathscr{F}\left(Y^{\sim}\right)(\bar{l})\right)=K_{\mathscr{F}(M)}\left(I_{M}(\bar{l}), \mathscr{F}\left(Y^{\sim}\right)(\bar{l})\right) .
$$

It implies $\left(Y^{C}\right) \sim \circ I_{M}=\mathscr{F}\left(Y^{\sim}\right)$.

Now we deduce immediately

$$
\left(Y^{(b)}\right) \sim I_{M}=b\left(\mathscr{F}\left(Y^{\sim}\right)\right) .
$$

Hence, by Propositions 5.3 (3) and 2.7 we obtain 


$$
\begin{aligned}
\left(\nabla_{X^{(a)}}^{C} Y^{(b)}\right) \sim I_{M} & =\left(X^{(a)}\right)^{\omega^{c}}\left(Y^{(b)}\right) \sim I_{M} \\
& =\left(X^{\omega}\right)^{(a)}\left(b \mathscr{F}\left(Y^{\sim}\right)\right) \\
& =b\left(X^{\omega}\right)^{(a)}\left(\mathscr{F}\left(Y^{\sim}\right)\right) \\
& =a b \mathscr{F}\left(X^{\omega}\left(Y^{\sim}\right)\right) \\
& =a b \mathscr{F}\left(\left(\nabla_{X} Y\right) \sim\right) \\
& =\left(\left(\nabla_{X} Y\right)^{(a b)}\right) \sim \circ I_{M} .
\end{aligned}
$$

This formula implies (5.13).

The uniqueness of $\nabla^{C}$ follows immediately from Corollary 2.8. The last part of the proposition is a consequence of (5.13).

The above theorem means that in the case of the tangent bundle $\mathscr{F}(M)=T M$ the constructed connection coincides with the connection defined by Yano and Kobayashi [32]. In the case of $r$-order tangent bundle, the tangent bundle of $p^{r}$-velocities, the tangent bundle of infinitesimal near points our construction coincides with the definition of Morimoto [18]-[23].

From Proposition 5.6 we can deduce formulas for Christoffel's symbols of $\nabla^{C}$. We will formulate these formulas in Section 7.

For torsions and curvatures of $\nabla$ and $\nabla^{C}$ we have

PROPOSITION 5.6. Let $\mathscr{F}$ be a product preserving functor. If $T$ and $R$ is the torsion and the curvature of $\nabla$, then $T^{C}$ and $R^{C}$ are the torsion and the curvature of $\nabla^{C}$.

Proof. Let $X, Y$ be vector fields on $M$ and $a, b$ be elements of the corresponding Weil algebra $A=\mathscr{F}(\mathbf{R})$. If $\tilde{T}$ is the torsion of $\nabla^{C}$, then from Proposition 5.6 and (2.15) we obtain

$$
\begin{aligned}
\tilde{T}\left(X^{(a)}, Y^{(b)}\right) & =\nabla_{X^{(a)}}^{C} Y^{(b)}-\nabla_{Y^{(b)}}^{C} X^{(a)}-\left[X^{(a)}, Y^{(b)}\right] \\
& =\left(\nabla_{X} Y\right)^{(a b)}-\left(\nabla_{Y} X\right)^{(a b)}-[X, Y]^{(a b)} \\
& =(T(X, Y))^{(a b)} \\
& =T^{C}\left(X^{(a)}, Y^{(b)}\right) .
\end{aligned}
$$

According to Proposition 2.9, $\tilde{T}=T^{c}$. Analogously we verify the formula for curvature tensors.

\section{Proposition 5.6 implies immediately}

COROLlary 5.8. $\quad \nabla$ is a torsionless connection if and only if so is $\nabla^{C} . \nabla$ is a curvatureless connection if and only if so is $\nabla^{C}$. 
We show the following proposition about relationships between geodesics of $\nabla$ and geodesics of $\nabla^{C}$.

Proposition 5.9. Let $\mathscr{F}$ be a product preserving functor. If $\gamma:(-\varepsilon,+\varepsilon) \rightarrow M$ is a geodesic of $\nabla$, then for each element $a$ of the corresponding Weil algebra $A=$ $\mathscr{F}(\mathbf{R})$ the curve $\gamma_{a}(t)=\mathscr{F}(\gamma)(t a)$ is a geodesic of $\nabla^{C}$.

Proof. Let $X$ be a local vector on $M$ such that $\dot{\gamma}(t)=X(\gamma(t))$.

Let $\varphi$ be a flow of $X$. Then $\gamma(t+s)=\varphi(t, \gamma(s))$ for sufficiently small $t, s$. Applying $\mathscr{F}$ we get $\mathscr{F}(\gamma)(a+b)=\mathscr{F}(\varphi)(a, \mathscr{F}(\gamma)(b))$ for $a, b$ in sufficient small neighborhood of $0 \in A$. Therefore, by Proposition 2.5, we obtain

$$
\begin{aligned}
\dot{\gamma}_{a}(t) & =\frac{d}{d s} \mathscr{F}(\gamma)((s+t) a)_{\mid s=0} \\
& =\frac{d}{d s} \mathscr{F}(\varphi)(s a, \mathscr{F}(\gamma)(t a))_{\mid s=0} \\
& =X^{(a)}\left(\gamma_{a}(t)\right) .
\end{aligned}
$$

Since $\left(\nabla_{X} X\right) \circ \gamma=0$, then

$$
\left(\nabla_{X} X\right)^{\left(a^{2}\right)} \circ \mathscr{F}(\gamma)=a^{2} \cdot \eta_{M} \circ \mathscr{F}\left(\left(\nabla_{X} X\right) \circ \gamma\right)=0
$$

Now, by (5.13) we have

$$
\begin{aligned}
\left(\nabla_{r_{a}}^{C} \dot{\gamma}_{a}\right)(t) & =\left(\nabla_{X^{(a)}}^{C} X^{(a)}\right)\left(\gamma_{a}(t)\right) \\
& =\left(\nabla_{X} X\right)^{\left(a^{2}\right)}\left(\gamma_{a}(t)\right) \\
& =0
\end{aligned}
$$

It means that $\gamma_{a}$ is a geodesic ${ }^{10)}$.

We can also prove

Proposition 5.10. Let $\mathscr{F}$ be a product preserving functor and $A=\mathscr{F}(\mathbf{R})$ be its Weil algebra. Let $\nabla$ be a connection of $M$ and $\gamma$ be its geodesic. If $X$ is a Jacobi field along $\gamma$ and $a \in A$, then $X_{a}(t)=\left(\eta_{M} \circ \mathscr{F}(X)\right)(t a)$ is a Jacobi field along $\gamma_{a}(t)=$ $\mathscr{F}(\gamma)(t a)$.

Proof. According to Proposition $5.9 \gamma_{a}$ is a geodesic on $\mathscr{F}(M)$ and if $Y$ is a local vector fields on $M$ such that $Y(\gamma(t))=\dot{\gamma}(t)$ then $Y^{(a)}\left(\gamma_{a}(t)\right)=\dot{\gamma}_{a}(t)$. Since

10) From the proof we deduce that if $a^{2}=0$, then $\gamma_{a}$ is geodesic on $\mathscr{F}(M)$ for every connection $\nabla$ on $M$ and every curve $\gamma$ on $M$. 
$X$ is a Jacobi field along $\gamma$ if and only if

$$
\nabla_{\dot{\gamma}}^{2} X+\nabla_{\dot{\gamma}} T(X, \dot{\gamma})+R(X, \dot{\gamma}) \dot{\gamma}=0,
$$

thus using the methods from Proposition 5.9 we can conclude our proposition.

We finish this section by the following proposition about covariant derivations.

Proposition 5.11. Let $\mathscr{F}$ be a product preserving functor, $A=\mathscr{F}(\mathbf{R})$ be its Weil algebra, $\nabla$ be a linear connection on a manifold $M, X$ be a vector field on $M$ and let $a$ $\in A$.

If $S$ is a tensor field of type $(1, k)$ on $M$ and $b \in A$, then

$$
\nabla_{X^{(a)}}^{C} S^{(b)}=\left(\nabla_{X} S\right)^{(a b)}
$$

If $G$ is a tensor field of type $(0, k)$ on $M$ and $\lambda: A \rightarrow \mathbf{R}$ is a linear function, then

$$
\nabla_{X^{(a)}}^{C} G^{(\lambda)}=\left(\nabla_{X} G\right)^{\left(\lambda \circ l_{a}\right)}
$$

Proof. Let $X_{1}, \ldots, X_{k}$ be vector fields on $M$ and $a_{1}, \ldots, a_{k} \in A$. Using

$$
\left(\nabla_{X} S\right)\left(X_{1}, \ldots, X_{k}\right)=\nabla_{X}\left(S\left(X_{1}, \ldots, X_{k}\right)\right)-\sum_{i=1}^{k} S\left(X_{1}, \ldots, \nabla_{X} X_{i}, \ldots, X_{k}\right)
$$

from (3.3) and (5.13) we obtain

$$
\begin{aligned}
\left(\nabla_{X^{(a)}}^{C} S^{(b)}\right)\left(X_{1}^{\left(a_{1}\right)}, \ldots, X_{k}^{\left(a_{k}\right)}\right)= & \nabla_{X^{(a)}}^{C}\left(S^{(b)}\left(X_{1}^{\left(a_{1}\right)}, \ldots, X_{k}^{\left(a_{k}\right)}\right)\right) \\
& -\sum_{i=1}^{k} S^{(b)}\left(X_{1}^{\left(a_{1}\right)}, \ldots, \nabla_{X^{(a)}}^{C} X_{i}^{\left(a_{i}\right)}, \ldots, X_{k}^{\left(a_{k}\right)}\right) \\
= & \nabla_{X^{(a)}}^{C}\left(S\left(X_{1}, \ldots, X_{k}\right)\right)^{\left(b a_{1} \ldots a_{k}\right)} \\
& -\sum_{i=1}^{k} S^{(b)}\left(X_{1}^{\left(a_{1}\right)}, \ldots,\left(\nabla_{X} X_{i}\right)^{\left(a_{i}\right)}, \ldots, X_{k}^{\left(a_{k}\right)}\right) \\
= & \left(\nabla_{X}\left(S\left(X_{1}, \ldots, X_{k}\right)\right)\right)^{\left(b a a_{1} \ldots a_{k}\right)} \\
& -\sum_{i=1}^{k}\left(S\left(X_{1}, \ldots, \nabla_{X} X_{i}, \ldots, X_{k}\right)^{\left(b a a_{1} \ldots a_{k}\right)}\right. \\
= & \left(\left(\nabla_{X} S\right)\left(X_{1}, \ldots, X_{k}\right)\right)^{\left(b a a_{1} \ldots a_{k}\right)} \\
= & \left(\nabla_{X} S\right)^{(a b)}\left(X_{1}^{\left(a_{1}\right)}, \ldots, X_{k}^{\left(a_{k}\right)}\right) .
\end{aligned}
$$

According to Proposition 2.9 the first part is verified.

A verification of the second part is similar. We use (4.3) instead of (3.3). 


\section{Lifts of Riemannian metrics and symplectic structures}

From propositions proved in Sections 4 and 5 we can deduce:

Proposition 6.1. Let $\mathscr{F}$ be a product preserving functor and $A=\mathscr{F}(\mathbf{R})$ be its Weil algebra. Let $\lambda: A \rightarrow \mathbf{R}$ be a linear function such that the symmetric form $A \times A$ $\ni(a, b) \rightarrow \lambda(a b) \in \mathbf{R}$ is non-singular and let $\left(p_{\lambda}^{+}, p_{\lambda}^{-}\right)$be its signature.

(1) If $g$ is a pseudo-Riemannian metric on $M$, then $g^{(\lambda)}$ is a pseudo-Riemannian metric on $\mathscr{F}(M)$. If $\left(p_{g}^{+}, p_{g}^{-}\right)$is the signature of $g$, then the signature of $g^{(\lambda)}$ is $\left(p_{g}^{+} p_{\lambda}^{+}\right.$ $\left.+\overline{p_{g}^{-}} p_{\lambda}^{-}, p_{g}^{+} p_{\lambda}^{-}+p_{g}^{-} p_{\lambda}^{+}\right)$.

(2) If $\nabla$ is the Riemannian connection of $g$, then $\nabla^{C}$ is the Riemannian connection of $g^{(\lambda)}$.

(3) If $X$ is a Killing vector field for $g$ and $a \in A$, then $X^{(a)}$ is a Killing vector field for $g^{(\lambda)}$

Proof. The part (1) is an immediate consequence of Proposition 4.5, the part (2) follows from Proposition 5.11 and the part (3) from Proposition 4.2 (4).

A Kählerian structure on a manifold $M$ is a couple $(g, J)$, where $g$ is a pseudo-Riemannian tensor on $M$ and $J$ is a complex structure on $M$ such that

$$
g(J X, Y)=-g(X, J Y), \quad \nabla_{X} J=0
$$

for all vector fields $X, Y$ on $M$, where $\nabla$ is the Riemannian connection of $g$. For Kählerian structures we have

THEOREM 6.2. Let $\mathscr{F}$ be a product preserving functor and $A=\mathscr{F}(\mathbf{R})$ be its Weil algebra. Let $\lambda: A \rightarrow \mathbf{R}$ be a linear function such that the symmetric form $A \times A \ni$ $(a, b) \rightarrow \lambda(a b) \in \mathbf{R}$ is non-singular. If $(g, J)$ is a Kählerian structure on $M$, then $\left(g^{(\lambda)}, J^{c}\right)$ is a Kählerian structure on $\mathscr{F}(M)$.

Proof. According to Proposition $6.1 \mathrm{~g}^{(\lambda)}$ is a pseudo-Riemannian metric on $\mathscr{F}(M)$ with Riemannian connection $\nabla^{C}$, where $\nabla$ is Riemannian connection of $g$, and by Theorem $3.5 J^{C}$ is a complex structure on $\mathscr{F}(M)$.

Using the notation of Section 4 the formula $g(J X, Y)=-g(X, J Y)$ can be written in the form $\rho^{1}(g, J)=-\rho^{2}(g, J)$. Using Propositions $4.2(3)$ and 5.11 we finish the proof. 
An almost symplectic structure on a manifold $M$ is a 2 -form $\omega$ on $M$ such that the mapping $T M \ni v \rightarrow i_{v} \omega \in T^{*} M$ is an isomorphism of vector bundles. The dimension of manifold $M$ on which there is an almost symplectic structure is odd. An almost symplectic structure $\omega$ on $M$ is called a symplectic structure if $d \omega=0$. We prove

Proposition 6.3. Let $\mathscr{F}$ be a product preserving functor and $A=\mathscr{F}(\mathbf{R})$ be its Weil algebra. Let $\lambda: A \rightarrow \mathbf{R}$ be a linear function such that the symmetric form $A \times A$ $\ni(a, b) \rightarrow \lambda(a b) \in \mathbf{R}$ is non-singular. If $\omega$ is an almost symplectic structure on $M$, then $\omega^{(\lambda)}$ is an almost symplectic structure on $\mathscr{F}(M)$.

If $\omega$ is a symplectic structure, then so is $\omega^{(\lambda)}$.

Proof. Let $\operatorname{dim} M=2 n$. For each point of $M$ there are a neighborhood $U$ and vector fields $X_{1}, \ldots, X_{n}, X_{n+1}, \ldots, X_{2 n}$ defined on $U$ such that (see [11] or [4])

$$
\omega\left(X_{\imath}, X_{j}\right)= \begin{cases}1, & \text { if } j=i+n, i=1, \ldots, n \\ -1, & \text { if } i=j+n, j=1, \ldots, n \\ 0, & \text { in the other cases. }\end{cases}
$$

Let $a_{1}, \ldots, a_{K}$ be a basis of $A$ such that

$$
\lambda\left(a_{\nu} a_{\mu}\right)= \begin{cases}1 & \text { for } \nu=\mu=1, \ldots, p \\ -1 & \text { for } \nu=\mu=p+1, \ldots, K \\ 0 & \text { in the other cases. }\end{cases}
$$

where $(p, K-p)$ is the signature of $A \times A \ni(a, b) \rightarrow \lambda(a b) \in \mathbf{R}$ and $K=\operatorname{dim} A$. Now, using (4.3) we compute the matrix $\Omega_{(i, \nu)(j, \mu)}$ of $\omega^{(\lambda)}$. We obtain

$$
\Omega_{(i, \nu)(j, \mu)}=\omega^{(\lambda)}\left(X_{i}^{\left(a_{\nu}\right)}, X_{j}^{\left(a_{\mu}\right)}\right)=\lambda\left(a_{\nu} a_{\mu} \mathscr{F}\left(\omega\left(X_{i}, X_{j}\right)\right) .\right.
$$

Since $\omega\left(X_{i}, X_{j}\right)$ is constant thus we have (cf. the proof of Proposition 4.5)

$$
\Omega_{(i, \nu)(j, \mu)}=\omega\left(X_{i}, X_{j}\right) \lambda\left(a_{\nu} a_{\mu}\right)= \begin{cases}1, & \text { if } j=i+n, \nu=\mu=1, \ldots, p \\ -1, & \text { if } j=i+n, \nu=\mu=p+1, \ldots, K \\ -1, & \text { if } i=j+n, \nu=\mu=1, \ldots, p \\ 1, & \text { if } i=j+n, \nu=\mu=p+1, \ldots, K \\ 0, & \text { in the other cases. }\end{cases}
$$

It means that the matrix $\Omega=\left[\Omega_{(i, \nu)(J, \mu)}\right]$ is a block-matrix of the form

$$
\Omega=\left[\begin{array}{cccc}
A_{1} & 0 & \cdots & 0 \\
0 & A_{2} & \cdots & 0 \\
\vdots & \vdots & \ddots & \vdots \\
0 & 0 & \cdots & A_{K}
\end{array}\right],
$$


where $p$ matrixes from $A_{1}, \ldots, A_{K}$ are equal to

$$
J=\left[\begin{array}{cc}
0 & I_{n} \\
-I_{n} & 0
\end{array}\right]
$$

and $K-p$ from this sequence are equal to $-J$. It implies that $\Omega$ is a nonsingular matrix. Thus, $\omega^{(\lambda)}$ is an almost symplectic structure on $\mathscr{F}(M)$.

The last part of the proposition follows from Corollary 4.4 .

Let $(g, J)$ be a Kählerian structure on $M$. Then $\omega(X, Y)=g(X, J Y)$ is a symplectic structure associated to the given Kählerian structure $(g, J)$. From Proposition 4.2 (3) we obtain immediately

Corollary 6.4. Let $\lambda: A \rightarrow \mathbf{R}$ be a linear function such that the symmetric form $A \times A \ni(a, b) \rightarrow \lambda(a b) \in \mathbf{R}$ is non-singular. If $\omega$ is a symplectic structure associated to a Kählerian structure $(g, J)$, then $\omega^{(\lambda)}$ is associated to $\left(g^{(\lambda)}, J^{C)}\right)$.

\section{Final remarks - local expressions}

We have proved all our theorems and propositions without using local expressions for lifted geometric objects. In the cases of the tangent bundle, the $r$-tangent bundle, the tangent bundle of $p^{r}$-velocities local expressions of coordinates of lifted objects were very important to prove main results. In the cases of these bundles local expressions have a nice and simple form because in the corresponding Weil algebras we can choose a basis with simple structure constants. In the general case formulas are more complicated.

Let $\mathscr{F}$ be a product preserving functor. In this section we fix a basis $a_{1}, \ldots$, $a_{K}$ of the Weil algebra $A=\mathscr{F}(\mathbf{R})$ associated to $\mathscr{F}$ and let $\alpha_{\nu \mu}^{\alpha}$ be the structural constants given by

$$
a_{\nu} a_{\mu}=\sum_{\kappa=1}^{K} \alpha_{\nu \mu}^{\varkappa} a_{\varkappa}
$$

We denote by $a_{1}^{*}, \ldots, a_{K}^{*}: A \rightarrow \mathbf{R}$ the dual basis.

We start our considerations from looking for a formula for $(f g)^{(\lambda)}$, where $f, g$ are functions on $M$ and $\lambda: A \rightarrow \mathbf{R}$ is a linear function. Using the formulas

$$
\mathscr{F}(f)=\sum_{\nu=1}^{K} f^{\left(a_{\nu}^{*}\right)} a_{\nu}, \quad \mathscr{F}(f g)=\mathscr{F}(f) \mathscr{F}(g)
$$

from the definition of $f^{(\lambda)}$ we conclude 


$$
\begin{aligned}
(f g)^{(\lambda)} & =\lambda \circ \mathscr{F}(f g)=\lambda \circ(\mathscr{F}(f) \mathscr{F}(g)) \\
& =\sum_{\nu, \mu=1}^{K} \lambda\left(f^{\left(a_{\nu}^{*}\right)} g^{\left(a_{\mu}^{*}\right)} a_{\nu} a_{\mu}\right. \\
& =\sum_{\nu, \mu, \kappa=1}^{K} \alpha_{\nu \mu}^{\alpha} \lambda\left(a_{\varkappa}\right) f^{\left(a_{\nu}^{*}\right)} g^{\left(a_{\mu}^{*}\right)} .
\end{aligned}
$$

Particularly, if $\lambda=\alpha_{\varkappa}^{*}$ is an element of the dual basis from (7.3) we obtain

$$
(f g)^{\left(a_{\kappa}^{*}\right)}=\sum_{\nu, \mu=1}^{K} \alpha_{\nu \mu}^{\chi} f_{\nu}^{\left(a_{\nu}^{*}\right)} g^{\left(a_{\mu}^{*}\right)} .
$$

If $f$ is a function on $M, X$ is a vector field on $M$ and $a \in A$, then from the equality $(f X)^{C}=\mathscr{F}(f) \cdot X^{C}$, the definition of $a$-lift of vector fields and from (7.2) we have

$$
\begin{aligned}
(f X)^{(a)} & =a \cdot(f X)^{c}=a \mathscr{F}(f) \cdot X^{C} \\
& =\sum_{\nu=1}^{K} f^{\left(a_{\nu}^{*}\right)} a a_{\nu} \cdot X^{C} \\
& =\sum_{\nu=1}^{K} f^{\left(a_{\nu}^{*}\right)} X^{\left(a a_{\nu}\right)} .
\end{aligned}
$$

Particularly, if $a=a_{\mu}$ is an element of the basis of $A$, then from (7.5) and (7.1) we obtain

$$
\begin{aligned}
(f X)^{\left(a_{\mu}\right)} & =\sum_{\nu=1}^{K} f^{\left(a_{\nu}^{*}\right)} X^{\left(a_{\nu} a_{\mu}\right)} \\
& =\sum_{\nu, \kappa=1}^{K} \alpha_{\nu \mu}^{\kappa} f^{\left(a_{\nu}^{*}\right)} X^{\left(a_{\kappa}\right)}
\end{aligned}
$$

Let $\left(U, x^{i}\right)$ be a chart on $M$. If $X=\sum_{i=1}^{n} X^{i} \partial_{i}$ is the local expression of a vector field $X$, then from (7.6) and Corollary 2.8 we obtain

$$
\begin{aligned}
X^{\left(a_{\nu}\right)} & =\sum_{i=1}^{n}\left(X^{i} \partial_{i}\right)^{\left(a_{\nu}\right)}=\sum_{i=1}^{n} \sum_{\mu, \kappa=1}^{K} \alpha_{\nu \mu}^{\kappa}\left(X^{i}\right)^{\left(a_{\mu}^{*}\right)} \partial_{i}^{\left(a_{\kappa}\right)} \\
& =\sum_{i=1}^{n} \sum_{\mu, \kappa=1}^{K} \alpha_{\nu \mu}^{\kappa}\left(X^{i}\right)^{\left(a_{\mu}^{*}\right)} \partial_{i, \kappa} .
\end{aligned}
$$

The above formula means that $\sum_{\mu=1}^{K} \alpha_{\nu \mu}^{\chi}\left(X^{i}\right)^{\left(a_{\mu}^{*}\right)}$ are coordinates of $X^{\left(a_{\nu}\right)}$.

Now using Propositions 3.1 and 4.1 we can calculate the local coordinates of $S^{(a)}$, where $S$ is a tensor fields of type $(1, k)$ or $(0, k)$.

To finish this section we prove a formula for the Christoffel' symbols of the complete lift of a linear connection $\nabla$. 
From Proposition 5.6, Corollary 2.8 and formulas (7.1), (7.6) and (7.7) we have

$$
\begin{aligned}
\nabla_{\partial_{i, \nu}}^{c} \partial_{j, \mu} & =\nabla_{\partial_{i}^{\left(a_{\nu}\right)}}^{c} \partial_{j}^{\left(a_{\mu}\right)}=\left(\nabla_{\partial_{i}} \partial_{j}\right)^{\left(a_{\nu} a_{\mu}\right)}=\sum_{k=1}^{n}\left(\Gamma_{i j}^{k} \partial_{k}\right)^{\left(a_{\nu} a_{\mu}\right)} \\
& =\sum_{k=1}^{n} \sum_{\rho=1}^{K} \alpha_{\nu \mu}^{\rho}\left(\Gamma_{i j}^{k} \partial_{k}\right)^{\left(a_{\rho}\right)} \\
& =\sum_{k=1}^{n} \sum_{\rho, x, \omega=1}^{K} \alpha_{\nu \mu}^{\rho} \alpha_{\rho \omega}^{\alpha}\left(\Gamma_{i j}^{k}\right)^{\left(a_{\omega}^{*}\right)} \partial_{k, \kappa} .
\end{aligned}
$$

Since the Christeffel' symbols $\Gamma_{(i, \nu),(j, \mu)}^{(k, x)}$ are defined by

$$
\nabla_{\partial_{i, \nu}}^{C} \partial_{j, \mu}=\sum_{k=1}^{n} \sum_{x=1}^{K} \Gamma_{(i, \nu),(j, \mu)}^{(k, \kappa)} \partial_{k, \kappa}
$$

thus from these two formulas we obtain

$$
\Gamma_{(i, \nu),(j, \omega)}^{(k, \kappa)}=\sum_{\rho, \omega=1}^{K} \alpha_{\nu \mu}^{\rho} \alpha_{\rho \omega}^{\alpha}\left(\Gamma_{i j}^{k}\right)^{\left(a_{\omega}^{*}\right)}
$$

\section{REFERENCES}

[1] L. A. Cordero, C. T. J. Dodson, M. de Leon, On the differential geometry of frame bundles, Kluwer, Dordrecht, 1989.

[2] R. Crittenden, Covariant differentiation, Quart. J. Math. Oxford, 13 (1963), 285-297.

[3] D. J. Eck, Product-preserving functors on smooth manifolds, J. Pure Appl. Algebra, 42 (1986), 133-140.

[4] J. Gancarzewicz, Complete lifts of tensor fields of type $(1, k)$ to natural bundles, Zeszyty Nauk. UJ, Kraków, 23 (1982), 43-79.

[5] J. Gancarzewicz, Differential Geometry (Polish), Monografie PWN, Warszawa 1987.

[6] J. Gancarzewicz, Prolongation of conjugate connections, Zeszyty Naukowe Politechniki Śląskiej, Gliwice, 64 (1990), 61-73.

[7] J. Gancarzewicz, S. Mahi, Géodésiques dans le fibré tangent d'ordre superieur, Univer. Iagellonicae Acta Matematica, XXVIII (1991), 233-243.

[8] J. Gancarzewicz, W. Mikulski, Z. Pogoda, Natural bundles and natural liftings. Prolongations of geometric structures, Proc. Conf. on Diff. Geometry and Its Applications, Opava, August 24-28, 1992, Silesian University, Opava (1993), 281-320, Ed. by O. Kowalski and D. Krupka.

[9] J. Gancarzewicz, W. Mikulski, Z. Pogoda, Properties of product preserving functors, Proc. Winter School on Diff. Geometry and Its Applications, Zdikov, January 1993 (to appear).

[10] C. Godbillon, Géometrie differentielle et méchanique, analitique, Paris 1969.

[11] G. Kainz, P. Michor, Natural transformations in differential geometry, Czech. Math. J., 37 (112), 1987, 584-607. 
[12] Kobayashi, K. Nomizu, Foundations of differencial geometry, vol. 1, Intersciences, New York 1963.

[13] I. Kolár̆, On natural operators on vector fields, Ann. Global Analysis and Geometry, 6(2) (1988), 109-117.

[14] I. Kolář, P. W. Michor, J. Slov́ak, Natural operations in differential geometry, Springer Verlag Berlin, 1993.

[15] I. Kolár̆, J. Slovák, On the geometric functors on manifolds, Suppl. ai Rendiconti del Cir. Math. di Palermo, 21 (1989), 223-233.

[16] O. O. Luciano, Categories of multiplicative functors and Morimoto's conjecture, Preprint 46, Inst. Fourier, Laboratoire des Mathématiques, Grenoble, 1986.

[17] W. Mikulski, Natural transformations trasforming functions and vector fields to functions on some natural bundles, Math. Bohemica, 117 (1992), 217-223.

[18] A. Morimoto, Prolongations of geometric structures, Lect. Notes Math. Inst. Nagoya Univ. 1969.

[19] - Prolongations of $G$-structures to tangent bundles, Nagoya Math. J., 32 (1968), 67-108.

[20] - Liftings of some type of tensor fields and connections to tangent bundles of $p^{r}$-velocities, Nagoya Math. J., 40 (1970), 13-31.

[21] - Prolongations of connections to tangential fibre bundles of higher order, Nagoya Math. J., 40 (1970), 85-97.

[22] - Liftings of tensor fields and connections to tangent bundles of higer order, Nagoya Math. J., 40 (1970), 99-120.

[23] - Prolongations of connections to bundles of infinitely near points, J. Diff. Geom., 11 (1976), 476-498.

[24] A. Nijenhuis, Natural bundles and their general properties, Diff. Geom. in honor of K. Yano, Tokyo 1972, 317-334.

[25] R. Palais and C.-L. Terng, Natural bundles have a finite order, Topology, 16 (1977), 271-277.

[26] Z. Pogoda, Liftings of connections with respect to $A$-functor, Demonstr. Math., (in press).

[27] S. E. Salvioli, Theory of geometric objects, J. Diff. Geom., 7 (1972), 257-278.

[28] J. Slovák, Prolongations of connections and sprays to Weil functors, Suppl. ai Reindicondi Circolo Math. Palermo, 14 (1987).

[29] A. Weil, Thèorie des points proches sur les variétés differentiables, Coloque Geom. Diff. Strasbourg 1953, 111-117.

[30] K. Yano and S. Ishihara, Tangent and cotangent bundles, Marcel Dekker, INC., New York, 1973.

[31] K. Yano, S. Kobayashi, Prolongations of tensor fields and connections to tangent bundles I., J. Math. Soc. Japan, 18 (1966), 194-210.

J. Gancarzewicz, W. Mikulski, Z. Pogoda Instytut Matematyki UJ

ul. Reymonta 4

30-059 Kraków, Poland e-mail:

(JG)gancarze@im.uj.edu.pl

(WM)mikluski@im.uj.edu.pl

(ZP)pogoda@im.um.edu.pl 